\title{
New rotation periods in the open cluster NGC 1039 (M 34), and a derivation of its gyrochronology age $e^{\star \star \star}$
}

\author{
D. J. James ${ }^{1,2}$, S. A. Barnes ${ }^{3}$, S. Meibom ${ }^{4}$, G. W. Lockwood ${ }^{3}$, S. E. Levine ${ }^{5}$, C. Deliyannis ${ }^{6}$, I. Platais ${ }^{7}$, \\ A. Steinhauer ${ }^{8}$, and B. K. Hurley ${ }^{1}$
}

${ }^{1}$ Hōkū Ke'a Observatory, Department of Physics \& Astronomy, University of Hawai'i at Hilo, 200 West Kawili Street, Hilo, HI 96720, USA e-mail: david.james@hawaii.edu

2 Department of Physics \& Astronomy, Vanderbilt University, Box 1807 Station B, Nashville, TN 37235, USA

3 Lowell Observatory, 1400 W. Mars Hill Rd., Flagstaff, AZ 86001, USA

4 Harvard-Smithsonian Center for Astrophysics, 60 Garden Street, Cambridge, MA 02138, USA

5 United States Naval Observatory, Flagstaff Station, 10391 West Naval Observatory Road, Flagstaff, AZ 86001-8521, USA

6 Astronomy Department, Indiana University, Swain Hall West 319, 727 East 3rd Street, Bloomington, IN 47405-7105, USA

7 Department of Physics \& Astronomy, Johns-Hopkins University, Baltimore, MD 21218, USA

8 Department of Physics and Astronomy, 1 College Circle, SUNY Geneseo, Geneseo, NY 14454, USA

Received 18 August 2009 / Accepted 16 March 2010

ABSTRACT

\begin{abstract}
Aims. Employing photometric rotation periods for solar-type stars in NGC 1039 [M34], a young, nearby open cluster, we use its mass-dependent rotation period distribution to derive the cluster's age in a distance independent way, i.e., the so-called gyrochronology method.

Methods. We present an analysis of 55 new rotation periods, using light curves derived from differential photometry, for solar type stars in the open cluster NGC 1039 [M34]. We also exploit the results of a recently-completed, standardized, homogeneous BVIc CCD survey of the cluster, performed by the Indiana Group of the WIYN open cluster survey, in order to establish photometric cluster membership and assign $B-V$ colours to each photometric variable. We describe a methodology for establishing the gyrochronology age for an ensemble of solar-type stars. Empirical relations between rotation period, photometric colour and stellar age (gyrochronology) are used to determine the age of M34. Based on its position in a colour-period diagram, each M34 member is designated as being either a solid-body rotator (interface or I-star), a differentially rotating star (convective or C-star) or an object which is in some transitory state in between the two (gap or g-star). Fitting the period and photometric colour of each I-sequence star in the cluster, we derive the cluster's mean gyrochronology age.

Results. Of the photometric variable stars in the cluster field, for which we derive a period, 47 out of 55 of them lie along the loci of the cluster main sequence in $V / B-V$ and $V / V-I$ space. We are further able to confirm kinematic membership of the cluster for half of the periodic variables [21/55], employing results from an on-going radial velocity survey of the cluster. For each cluster member identified as an I-sequence object in the colour-period diagram, we derive its individual gyrochronology age, where the mean gyro age of M34 is found to be $193 \pm 9$ Myr.

Conclusions. Using differential photometry, members of a young open cluster can be easily identified in a colour-magnitude diagram from their periodic photometric variability alone. Such periodicity can be used to establish a period-colour distribution for the cluster, which for M34, we have used to derive its gyrochronology age of $193 \pm 9$ Myr. Formally, our gyro age of M 34 is consistent (within the errors) with that derived using several distance-dependent, photometric isochrone methods (250 $\pm 67 \mathrm{Myr})$.
\end{abstract}

Key words. methods: data analysis - starspots - stars: fundamental parameters - globular clusters: individual: NGC 1039 (M 34 )

\section{Introduction}

Over the past century, as instrumentation and detector technology have advanced, the methods by which stellar age is determined have also evolved. While we still rely heavily upon the use of theoretical model isochrones, fitting empirical luminositytemperature data (on an H-R diagram - e.g., Sandage 1958;

* Appendices A-C are only available in electronic form at http: //www . aanda. org

$\star \star$ Data of Appendices $\mathrm{A}-\mathrm{C}$ are only available in electronic form at the CDS via anonymous ftp to cdsarc.u-strasbg.fr $(130.79 .128 .5)$ or via

http://cdsweb.u-strasbg.fr/cgi-bin/qcat?J/A+A/515/A100
Demarque \& Larson 1964; Demarque \& Gisler 1975; Mengel et al. 1979; and more latterly, Meynet et al. 1993; D'Antona \& Mazzitelli 1997; Naylor 2009), several alternative techniques for determining stellar age now exist which exploit a more diverse assembly of fundamental stellar properties, such as magnetic activity, elemental abundances, white dwarf cooling time-scales and angular momentum content.

Inspired by the pioneering efforts of Wilson (1963), observations of solar type stars have shown that there is a clear age dependence in the strength of magnetic activity indicators (e.g., Ca II H \& K and X-rays), whose emissions are presumably due to solar-like magnetic field activity (e.g., Wilson 1964; Wilson \& Skumanich 1964; Wilson 1966; Skumanich 1972; 
Barry et al. 1981; Noyes et al. 1984; Maggio et al. 1987; Soderblom et al. 1991; Henry et al. 1996; Mamajek \& Hillenbrand 2008). While there is not a one-to-one relationship between activity and age for any given star, current data samples unequivocally show there to be a strong correlation between decreasing magnetic activity and increasing age.

However, even such a well-observed phenomenon does not deliver unequivocal results, as illustrated for instance by the factor of two range in ages derived by Giampapa et al. (2006) for photometric and kinematic members of the 4 Gyr-old open cluster M 67. Moreover, stellar activity cycles, akin to those occurring in the Sun, can also lead to difficulties in establishing ages for individual stars. For instance, Soderblom et al. (1991) show that errors on chromospheric ages are roughly 50\%. Presumably, this error could be driven down statistically in a cluster by measuring many stars, and by removing close binaries or other contaminants. Soderblom et al. (2001) have provided chromospheric $\mathrm{H} \alpha$ measurements for a significant number of M 34 stars, but to our knowledge, a chromospheric age for M 34 has not yet been published.

More recently, age determinations for young open clusters have been advanced based on an analysis of their light element abundance distributions, specifically using lithium as a tracer. This technique, the so-called lithium depletion boundary method (Basri et al. 1996; Rebolo et al. 1996; Stauffer et al. 1998; Barrado y Navascués et al. 1999), does however have both merits and limitations. Line strengths and elemental abundance ratios are fairly straight forward to measure, and are indeed fundamental stellar properties, perhaps more so than compared to magnetic activity indicators. Unfortunately, the strength of this technique lies in detecting lithium in M-stars and cooler, which for any group of stars at a given distance are among the faintest. Even for nearby open clusters, detecting the lithium boundary thus requires use of precious 8-10 m class telescope time. Perhaps the ultimate limitation for this technique is that it is only discernibly sensitive to stars of $\$ 250$ Myr (e.g., Stauffer et al. 1998), severely limiting its widespread applicability.

The use of heavy-element abundances to establish stellar age has also been attempted, however its implementation is as yet limited. Cayrel et al. (2001) advocate using the detection of the singly-ionized uranium 238 line at $3859.6 \AA$ as an age proxy, although as they themselves note, the accuracy of this technique is currently limited by deficiencies in the nuclear data e.g., poorly-known oscillator strengths. Even with the advent of well-calibrated atomic data for uranium species, dating of open clusters using detection of stellar uranium will only be feasible for clusters older than the Hyades [600 Myr], due to the prohibitively long half-lives of the two dominant naturally occurring uranium species $\left(\tau_{1 / 2}=7.04 \times 10^{8}\right.$ and $4.47 \times 10^{9}$ yrs for $U^{235}$ and $U^{238}$ respectively - CRC 2005).

Spectroscopic and photometric observations of white dwarfs, in the Galactic field and in globular and open clusters, can also yield an age estimate of the their stellar content. The detailed physics of how white dwarfs cool as they age is now well developed (Mestel 1952; Cox \& Giuli 1965; Beaudet et al. 1967), and their model-dependent cooling time-scales are well described using theoretical stellar models (Koester \& Schönberner 1986; D’Antona \& Mazzitelli 1989; Iben \& Laughlin 1989; Wood 1992; Fontaine et al. 2001). This technique has been historically rather difficult to implement in open clusters. First, even for the nearby clusters, their white dwarf members are the faintest objects comprising the mass function, typically being fainter than $V=20$ th mag (e.g., Rubin et al. 2008), which is challenging for all but the largest aperture terrestrial telescopes. Second, white dwarfs are intrinsically blue objects (typically $B-V_{o}<0.2$ ), which until recently was problematic for capturing photons efficiently using astronomical, red sensitive spectrographs and detectors. Third, and most importantly, to properly derive the age of the cluster one must observe and characterize objects extending right down to the bottom of the white dwarf sequence; i.e., those objects which are the dimmest, the coolest and hence the oldest white dwarfs in the cluster. Consequently, in light of such procedural difficulties, relatively few open clusters have white dwarf cooling-time age estimates available in the literature (e.g., Richer et al. 1998; Bedin et al. 2005; Kalirai et al. 2003, 2005; Rubin et al. 2008).

In terms of rotation, Kawaler (1989) uses theoretical stellar spin-down models (from Kawaler 1988) to analytically derive a relationship uniquely connecting a star's $B-V$ photometric colour and age to its period. Using a photometric colour, rotation period dataset for $\mathrm{G} \& \mathrm{~K}$-stars in the Hyades open cluster, he shows the Hyades rotation age to be $4.9 \pm 1.1 \times 10^{8}$ years. More recently, Barnes (2003, 2007 - hereafter B03, B07 respectively) exploit the morphology of photometric colour-rotation period distributions in coëval samples of stars (e.g., open clusters and binary systems) to establish stellar age. In essence, this so-called gyrochronology technique allows one to construct rotational isochrones, in order to trace the boundaries of age dependent colour-period distributions. Crucially however, these boundary definitions further suggest the identification and coupling-state of each star's basic internal stratification in terms of their radiative zone and convection envelope.

Solar-type stars lying close to or on the I, or interface sequence, are probably rotating as solid bodies or close to it, and their spin-down evolution is Skumanich-like (Skumanich 1972), i.e., directly proportional to the square root of stellar age (spindown $\propto t^{1 / 2}$ ). The most rapidly rotating solar-type stars at a given mass lie on or close to the $\mathrm{C}$, or convective sequence. These stars are thought to be rotationally de-coupled; that is to say, it is likely that only their outer convective envelope is spinning down, with an exponential time dependency (spin-down $\propto e^{f(t)}$ ). Early incarnations of rotational evolution models incorporating de-coupled stellar structure were referred to as core-envelope (de)coupling models (cf., Endal \& Sofia 1981; Stauffer et al. 1984; Soderblom et al. 1993a; Jianke \& Collier Cameron 1993). Those stars which are in a transitory state between the $\mathrm{C}$ and I-sequences, representing a scenario whereby the outer convective envelope is re-coupling, probably magneto-hydrodynamically, to the inner radiative zone, constitute the so-called gap or g-stars in the gyrochronology paradigm.

Establishing stellar age for open clusters using gyrochronology models of course has its limitations. For instance for open clusters, enough stars must be photometrically monitored in order to derive rotation periods, so that a clear, well-defined distribution of interface, gap, or convective sequence objects is apparent. Such an observing programme typically requires extensive allocations of telescope time and considerable human effort. Moreover, differential rotation of solar-type stars and multiple star-spot groups on their surfaces can also introduce ambiguities into gyrochronology analyzes, since it can act to smear-out the distribution of rotation periods of a given mass.

For small samples of rotation periods in open clusters, the gyrochronology method is not applicable in multiple systems where other effects might interfere with, or even overwhelm, the regular wind-related loss of angular momentum. For instance, close binaries ( $a \lesssim 0.1 \mathrm{AU}$ ) experience a tidal torque which acts to synchronize their rotation with the orbital motion of the system, in addition to any magnetic torque they 
experience due to stellar winds (Zahn 1989; 1994); thus, as they evolve along the main-sequence, components in the closest binaries tend to rotate faster (on average) than single stars of similar mass and age. Observations of RS CVn and BY Dra systems have shown Zahn's theoretical framework to be correct (Hall 1976; Bopp \& Fekel 1977; Fekel et al. 1986). Binary/multiple systems must therefore be avoided, where the data allow, in establishing gyrochronology ages of stars (see Meibom et al. 2006, for a more thorough discussion).

A recent International Astronomical Union symposium (IAUS 258), dedicated to the subject of The Age of Stars, now provides our community with a thorough overview of an historical and modern approach to understanding the how and why of stellar ages. A careful perusal of the symposium proceedings allows, for both the novice and expert alike, a detailed insight into the theoretical framework unpinning stellar age determinations as well as the observational data calibrating and constraining such models. Of special interest to this manuscript were the oral presentations by Barnes (2009), Jeffries (2009) and Meibom (2009).

The genesis of this gyrochronology project for M34, $\mathrm{a} \simeq 200$ Myr open cluster, occurred nearly ten years ago. At that epoch, SAB and GWL obtained a differential photometry dataset at Lowell Observatory over 17-nights for the Western half of the cluster, with the goal of deriving rotation periods for photometric variables in the field. The project lay dormant for some years until we noticed that Irwin et al. (2006) published new rotation period data for a sample of solar-type stars in the cluster. Unfortunately in terms of rotation, there are several fundamental problems with the Irwin et al. dataset which hamper our ability to pursue a rigorous gyrochronology analysis using their data (see Sect. 4.4 for details). A natural progression and indeed amelioration of their period distribution analysis is to derive the gyrochronology properties of this cluster, now incorporating rotation periods derived from the differential photometry that we had obtained during the 1998 Lowell campaign. In this manuscript therefore, we now report an analysis of our differential photometry dataset for the cluster.

New BVI photometry is reported in Sect. 3 for each photometric variable discovered in our Lowell observing campaign. Rotation periods derived from light-curve analysis of the photometric variables in our field of view are described in Sect. 4, which includes a comparison with the Irwin et al. study. Using a clean sample of rotation periods for photometric and/or kinematic members of the M34 cluster, we describe in Sect. 5 how colour-period data are used in undertaking a gyrochronology analysis, which results in the derivation of the rotation age of the cluster. Also included is a commentary on how error in the gyrochronology age can be ascribed to disparate physical properties of the colour-period dataset, such as cluster nonmembership, binarity and differential rotation.

\section{The open cluster Messier 34}

M 34 (NGC 1039) is a young ( $\simeq 200$ Myr; see Table 1$)$ open cluster on the border of Perseus and Andromeda [RA(2000): $02^{\mathrm{h}} 42^{\mathrm{m}}$, Dec(2000): $\left.+42^{\circ} 46^{\prime}\right]$. It is $\simeq 470 \mathrm{pc}$ away, out of the Galactic plane $\left[b \simeq-16^{\circ}\right.$, and has a reddening of $E_{B-V}=0.07$ (Canterna et al. 1979). These characteristics suggested to us that it would make a good target for rotation period determinations, including a derivation of its rotational age via the methodology of gyrochronology.
Table 1. Extant isochronal ages for M 34

\begin{tabular}{crr}
\hline \hline Method & $\begin{array}{r}\text { Stellar Age } \\
{[\mathrm{Myr}]}\end{array}$ & Reference \\
\hline$U B V$ & 100 & Cester et al. (1977) \\
$u v b y$ & 500 & Canterna et al. (1979) \\
$U B V$ & 250 & Ianna \& Schlemmer (1993) \\
$U B V$ & 180 & Meynet et al. (1993) \\
$B V I c$ & $200-250$ & Jones \& Prosser (1996) \\
\hline
\end{tabular}

\subsection{Extant observations of M34}

Prior work on M 34 includes photoelectric $U B V$ observations for 57 stars bluer than F3 in the M 34 field by Johnson (1954) and of 43 similar stars by Cester et al. (1977). Canterna et al. (1979) reported photoelectric photometry in Strömgren $u v b y$ and $\mathrm{H} \beta$, for some 42 M 34 stars. Jones \& Prosser (1996) published a more extensive and deeper (down to $V=16.2$ ) survey using $B V I$ CCD observations. An even deeper $(V \simeq 24) V I$ CCD survey has been recently published by Irwin et al. (2006). Both of the latter two studies have certain irregularities with the standardization procedures that are evident in the papers. Consequently, we use standardized photometry from a new $B V R I$ survey by the Indiana group of the WIYN open cluster study (Deliyannis et al., in prep.) in our analysis (see Sect. 3).

Two proper motion surveys have been conducted in M34. The first, by Ianna \& Schlemmer (1993), has a limiting magnitude of $V=14.5$, and the second, by Jones \& Prosser (1996), one of $V=16.2$. Reliable membership probabilities from these studies are limited to stars brighter than $V=13.5$ and $V=14.5$, respectively, and although $>80 \%$ of the stars in our study are fainter than either of the proper motion surveys, we use them where available. Jones et al. (1997) have provided radial velocities for some 47 cluster members but few of these overlap with our photometric period sample. However, we take recourse to an on-going multi-epoch WIYN HYDRA radial velocity survey of the cluster (Meibom et al., in prep.) to provide a reliable kinematic membership criterion for nearly half $(25 / 55)$ of the M34 stars for which we report new photometric periods. A thorough discussion of extant membership probabilities for M34 stars comprising our gyrochronology sample, as well as new high resolution spectroscopic results for two photometric variables in our sample, is presented in Appendix A.

Other meritorious work on M 34 includes a study of the cluster white dwarfs by Rubin et al. (2008), who derive a minimum age of the cluster to be $64 \mathrm{Myr}$. Although Rubin et al. were not explicitly attempting an age derivation, such a result must be considered with some caution because of the lack of surety of cluster membership for their targets, the small sample size ( $5 \mathrm{ob}-$ jects), and the fact that only the very top of the cluster white dwarf sequence was sampled, whereby the coolest, and hence oldest white dwarfs in the cluster were not observed.

Projected equatorial rotation velocities $[v \sin i]$ for the earlier-type, higher mass stars in M 34 are provided by Ianna (1970), Hartoog (1977), and Landstreet et al. (2008), whereas for its solar-type members velocities are published by Jones et al. (1997) and Soderblom et al. (2001). For stars in common, we provide a comparison between these $v \sin i$ data and our new photometric periods in Appendix B. By removing the dual ambiguities in $v \sin i$ data (the obvious $\sin i$ factor, and the stellar radius), photometric rotation period studies far and above supersede $v \sin i$ studies - provided of course that the rotation periods are measured correctly. 
Irwin et al. (2006) have provided rotation periods for some 105 stars in M34, using photometric data obtained under considerably more restrictive conditions (observing window, and filters) than ours. Although half the stars in common between their study and our Lowell sample yield essentially the same period from multiple time-series, some discrepancies exist. A detailed comparison between their periods and ours is presented in Sect. 4.4.

\section{New Indiana CCD photometry}

A new, comprehensive BVRI survey of star fields in the direction of the M34 cluster is now available from the Indiana Group of the WIYN Open Cluster Survey (Deliyannis et al., in prep). Photometric observations of the central $40^{\prime} \times 40^{\prime}$ of the cluster, including $\simeq 100$ Landolt (1992) standard stars, were obtained using the WIYN 0.9-m telescope during the night of 24-Oct.-1998. The $2048 \times 2048$ pixel T2KA Tekronics CCD was employed at the $f / 7.5$ Cassegrain focus, which with its $0^{\prime}$.68 pixel $^{-1}$ plate scale yields an effective field of view of $\simeq 23^{\prime} \times 23^{\prime}$. Multiple exposures in each filter were obtained over a $2 \times 2$ mosaic for the cluster. Images were analyzed using the dedicated DAOPHOT II photometry data reduction package. Landolt standard star frames were processed using aperture photometry, whereas object frames were fitted with spatiallyvariable point spread functions [PSFs], and spatially-variable aperture corrections. Both the PSFs and aperture corrections were determined empirically using hundreds of isolated stars on each frame. External errors in placing instrumental magnitudes onto the standard system are $<0.02 \mathrm{mag}$.

The results of the Indiana Group's photometric survey for stars relevant to this study are presented in Table 2, as well as coordinates for each star derived from SuperCosmos survey images (Hambly et al. 2001a-c). We also provide a kinematic membership assessment from an on-going survey of the cluster (Meibom et al., in prep.). For two M 34 targets for which we derive a period, F4_1925 and F4_2226, there are no $B$-band photometric data available. In this instance, we rely upon a field star $V-I$ to $B-V$ relationship (Caldwell et al. 1993), and an assumed $E_{B-V}=0.07$ (Canterna et al. 1979), to transform these stars' $V-I c$ colours into $B-V$ ones.

\section{New photometric periods in M 34}

\subsection{Observational programme}

We initiated a differential photometry programme of observations for solar-type stars in M34 during SeptemberOctober 1998, consisting of four separate sequences of CCD images in the $V$ and $I$ filters, acquired respectively during a 17 night observing run (UT980929 through 981015) at Lowell Observatory, Flagstaff, Arizona, USA. The dataset consists of $\sim 60$ visitations to each of two $19^{\prime} \times 19^{\prime}$ fields of the Western region of M34. Each visitation typically consisted of short $(60,40 \mathrm{~s})$ and long $(600,400 \mathrm{~s})$ exposures in the $V$ and $I$ filters respectively, using the $2048 \times 2048$ SITe chip mounted at the Cassegrain focus of the 42inch Hall telescope of Lowell Observatory. Each field received 4-7 visitations per night in each filter, over the first 12 nights, barring UT981008 which was lost to clouds, and one visitation each on the remaining 5 nights. The time-series analysis is performed on the instrumental system with respect to chosen reference images on UT981001.

\subsection{Data analysis and time series analysis}

The individual images were de-biased and flat-fielded using standard procedures in the IRAF $^{1}$ image reduction utility. Photometry for all objects on the processed images was computed via PSF fitting using DAOPHOT II and ALLSTAR II (Stetson et al. 1991). Typically, we have used 50 PSF stars per image and a quadratically-varying PSF. The photometry from the individual frames was cross-matched and converted into time series using the DAOMATCH/DAOMASTER routines from Stetson (1992). These routines match stars appearing in multiple images according to user-supplied specifications, calculate the photometric offsets between frames, and output the corrected time series. The uncertainty in the frame-to-frame magnitude offsets was less than $0.001 \mathrm{mag}$ for the frames that were eventually used because of the large number $(\gtrsim 1000)$ of stars matched from frame to frame. This procedure left us with totals of $\sim 4000, \sim 3000, \sim 2700$, and $\sim 2200$ objects respectively with time series in each of the long-I, long-V, short-I and short-V exposures for the western M 34 fields.

We tested every object in the field for variability without regard to its position in a colour-magnitude diagram, or any other consideration except its intrinsic variability. Also, we performed the variability/periodicity analysis independently in each exposure time/filter combination, in order to confirm or deny it, and to calculate period error. The following procedure was adopted for each time-series file. The time series files for each field were sigma-clipped to eliminate outliers ${ }^{2}$, and then we calculated a reduced chi square $\left(\tilde{\chi}^{2}\right)$ for each time-series, to glean out the most variable objects. Every object with $\tilde{\chi}^{2}>3$ was subjected to a periodicity search using the CLEAN algorithm of Roberts et al. (1987). The results were examined visually, and phased about reliable peaks in the power spectrum to yield phased light curves for the periodic variables. Finally, results of the (up to) four independent time-series analyzes on each object were merged together to confirm or deny the periodicity. For these stars, we calculate period errors based on multiple measurements.

As a result of the foregoing analysis, we have been able to identify forty-seven (47) objects with periods confirmed in at least two filter, exposure combinations. In addition, we derive periods for eight (8) objects for which we can only detect periodicity in one filter/exposure. The period analysis results are listed in Table 3, which also contains details of their derived periods, and standard deviations of magnitude variation in each timeseries. Stars having only one period determination are assigned period errors based on the widths of the peaks in the power spectra. Phased photometric light-curves, on the instrumental system, are presented for each of our M 34 variables in Appendix C.

Our 17-night observing window allows us to detect two or more phases for all periods up to about 8.5 days. However this cut is probably too conservative, and because the slow rotators are of great interest to us, we extend our analysis to periods longer than 8.5 days. We are confident about periods as long as three quarters of the $17 \mathrm{~d}$ baseline, i.e. $\sim 13 \mathrm{~d}$; however, the period errors are larger for such stars. For instance, F3_0469 (field 3) has a period of $\sim 12.9 \mathrm{~d}$ in all 4 exposure time/filter

\footnotetext{
1 IRAF is distributed by the National Optical Astronomy Observatories, which are operated by the Association of Universities for Research in Astronomy, Inc., under cooperative agreement with the National Science Foundation.

2 This procedure lowers our sensitivity to eclipsing binaries and flares or other one-time variables, but has the virtue of eliminating occasional bad photometric points, which could reduce the efficacy of the periodsearching software for rotational variables.
} 
D. J. James et al.: The gyrochronology age of M 34

Table 2. Astrometry, photometry and membership assignments are presented for those M 34 stars for which photometric periods are derived in the Lowell campaign.

\begin{tabular}{|c|c|c|c|c|c|c|c|c|c|c|c|}
\hline $\begin{array}{l}\text { Internal }^{a} \\
\text { Identifier }\end{array}$ & $\begin{array}{c}\mathrm{RA}^{b} \\
(\mathrm{~J} 2000)\end{array}$ & $\begin{array}{c}\operatorname{Dec}^{b} \\
(\mathrm{~J} 2000)\end{array}$ & $\mathrm{JP} 96^{c}$ & $V^{d}$ & $\operatorname{Verr}^{d}$ & $B-V^{d}$ & $B$-Verr ${ }^{d}$ & $V-I^{d}$ & $V$-Ierr $^{d}$ & $\begin{array}{l}\text { Phot }^{e} \\
\text { Mem }\end{array}$ & $\begin{array}{l}\mathrm{RV}^{e} \\
\text { Mem }\end{array}$ \\
\hline F3_0172 & 024105.141 & +425643.202 & JP 49 & 14.584 & 0.017 & 0.881 & 0.023 & 0.956 & 0.024 & Yes & Yes-sing \\
\hline F3_0176 & 024136.611 & +425020.288 & JP 167 & 14.659 & 0.009 & 0.888 & 0.013 & 1.051 & 0.013 & Yes & Yes-sing \\
\hline F3_0215 & 024100.981 & +425246.695 & JP 41 & 14.918 & 0.010 & 0.944 & 0.015 & 1.039 & 0.014 & Yes & Yes-sing \\
\hline F3_0258 & 024202.552 & +425151.721 & JP 289 & 15.236 & 0.009 & 1.017 & 0.013 & 1.118 & 0.013 & Yes & Yes-sing \\
\hline F3_0306 & 024157.472 & +4300 26.530 & JP 265 & 15.503 & 0.014 & 1.061 & 0.019 & 1.262 & 0.027 & Yes & Yes-bin \\
\hline F3_0320 & 024137.557 & +425721.593 & JP 172 & 15.664 & 0.017 & 1.116 & 0.025 & 1.237 & 0.024 & Yes & \\
\hline F3_0430 & 024150.490 & +425810.016 & & 16.424 & 0.013 & 1.309 & 0.019 & 1.505 & 0.018 & Yes & Yes-sing \\
\hline F3_0469 & 024049.090 & +424820.648 & & 16.604 & 0.018 & 1.353 & 0.030 & 1.550 & 0.025 & Yes & \\
\hline F3_0485 & 024106.638 & +424822.011 & & 16.732 & 0.010 & 1.355 & 0.016 & 1.619 & 0.014 & Yes & \\
\hline F3_0487 & 024044.889 & +425347.221 & & 16.818 & 0.020 & 1.348 & 0.030 & 1.701 & 0.027 & Yes & \\
\hline F3_0600 & 024118.447 & +425821.342 & & 17.394 & 0.020 & 1.522 & 0.035 & 1.863 & 0.027 & Yes & \\
\hline F3_0664 & 024120.454 & +425852.278 & & 17.862 & 0.022 & 1.511 & 0.057 & 1.999 & 0.029 & Yes & \\
\hline F4_0136 & 024147.384 & +424338.525 & JP 213 & 13.151 & 0.010 & 0.614 & 0.014 & 0.718 & 0.015 & Yes & Yes-sing \\
\hline F4_0169 & 024104.952 & +424651.343 & JP 50 & 13.510 & 0.010 & 0.668 & 0.014 & 0.786 & 0.014 & Yes & Yes-sing \\
\hline F4_0234 & 024133.429 & +424211.625 & JP 148 & 13.959 & 0.010 & 0.768 & 0.014 & 0.844 & 0.014 & Yes & Yes-sing \\
\hline F4_0303 & 024149.932 & +423713.906 & JP 227 & 14.366 & 0.009 & 0.837 & 0.013 & 0.912 & 0.013 & Yes & Yes-sing \\
\hline F4_0317 & 024149.354 & +423637.613 & JP 224 & 14.410 & 0.010 & 0.875 & 0.014 & 0.959 & 0.014 & Yes & Yes-sing \\
\hline F4_0327 & 024144.167 & +424607.333 & JP 199 & 14.483 & 0.009 & 0.883 & 0.013 & 0.941 & 0.013 & Yes & Yes-sing \\
\hline F4_0335 & 024135.247 & +424102.323 & JP 158 & 14.546 & 0.010 & 0.860 & 0.014 & 0.994 & 0.014 & Yes & Yes-bin \\
\hline F4_0450 & 024123.098 & +424015.954 & JP 113 & 14.960 & 0.010 & 0.965 & 0.014 & 1.058 & 0.014 & Yes & Yes-sing \\
\hline F4_0667 & 024106.187 & +424620.783 & JP 52 & 15.623 & 0.009 & 1.100 & 0.013 & 1.233 & 0.013 & Yes & Yes-bin \\
\hline F4_0695 & 024142.922 & +423313.910 & JP 197 & 15.643 & 0.021 & 1.156 & 0.030 & 1.289 & 0.029 & Yes & Yes-sing \\
\hline F4_0730 & 024049.647 & +424654.799 & JP 18 & 15.741 & 0.017 & 1.121 & 0.025 & 1.257 & 0.024 & Yes & \\
\hline F4_0736 & 024048.542 & +423925.716 & & 15.844 & 0.021 & 1.078 & 0.028 & 1.328 & 0.029 & Yes & Yes-bin \\
\hline F4_0803 & 024150.301 & +424437.371 & JP 229 & 16.025 & 0.010 & 1.234 & 0.014 & 1.373 & 0.013 & Yes & Yes-sing \\
\hline F4_0804 & 024148.974 & +423959.680 & & 16.034 & 0.010 & 1.176 & 0.015 & 1.378 & 0.014 & Yes & \\
\hline F4_0852 & 024153.246 & +423526.394 & & 16.294 & 0.009 & 1.225 & 0.013 & 1.634 & 0.013 & Yes & Yes-bin \\
\hline F4_0942 & 024146.371 & +423232.128 & & 16.341 & 0.020 & 1.268 & 0.029 & 1.498 & 0.028 & Yes & \\
\hline F4_1007 & 024050.752 & +424119.844 & & 16.839 & 0.022 & 1.510 & 0.033 & 1.901 & 0.034 & Yes & \\
\hline F4_1020 & 024036.693 & +424132.500 & & 16.608 & 0.021 & 1.330 & 0.031 & 1.584 & 0.030 & Yes & \\
\hline F4_1315 & 024053.997 & +423848.513 & & 17.347 & 0.022 & 1.441 & 0.036 & 1.815 & 0.030 & Yes & \\
\hline F4_1357 & 024144.198 & +423536.074 & & 17.476 & 0.011 & 1.450 & 0.021 & 1.911 & 0.015 & Yes & \\
\hline F4_1369 & 024151.600 & +422950.864 & & 17.496 & 0.015 & 1.513 & 0.027 & 1.934 & 0.021 & Yes & \\
\hline F4_1392 & 024138.851 & +424301.148 & & 17.654 & 0.011 & 1.504 & 0.025 & 1.963 & 0.015 & Yes & \\
\hline F4_1566 & 024131.131 & +424114.283 & & 18.132 & 0.012 & 1.549 & 0.032 & 2.137 & 0.016 & Yes & \\
\hline F4_1617 & 024126.292 & +423015.124 & & 18.165 & 0.027 & 1.570 & 0.064 & 2.178 & 0.035 & Yes & \\
\hline F4_1793 & 024120.042 & +423923.652 & & 18.606 & 0.015 & 1.393 & 0.063 & 2.285 & 0.019 & Yes & \\
\hline $\mathrm{F} 4 \_1925^{f}$ & 024121.947 & +423232.637 & & 19.040 & 0.037 & 1.577 & 0.045 & 2.350 & 0.045 & Yes & \\
\hline $\mathrm{F} 4 \_2226^{f}$ & 024031.108 & +424025.105 & & 19.659 & 0.067 & 1.625 & 0.071 & 2.626 & 0.071 & Yes & \\
\hline F3_0071 & 024044.422 & +425404.516 & & 12.855 & 0.019 & 1.047 & 0.025 & 1.153 & 0.026 & $\mathrm{No}$ & \\
\hline F3_0121 & 024147.414 & +425009.223 & JP 212 & 13.741 & 0.009 & 1.248 & 0.013 & 1.354 & 0.013 & No & \\
\hline F3_0163 & 024155.003 & +425253.485 & JP 258 & 14.440 & 0.008 & 0.900 & 0.011 & 0.986 & 0.012 & Yes & No \\
\hline F3_0165 & 024151.794 & +424620.473 & JP 244 & 14.419 & 0.008 & 0.865 & 0.011 & 0.956 & 0.011 & Yes & No \\
\hline F3_0388 & 024138.227 & +425543.112 & JP 174 & 15.952 & 0.010 & 0.975 & 0.015 & 1.076 & 0.022 & No & \\
\hline F3_0413 & 024148.478 & +424933.840 & JP 218 & 16.242 & 0.010 & 1.143 & 0.015 & 1.412 & 0.014 & Yes & No \\
\hline F3_0437 & 024048.970 & +425612.434 & & 16.022 & 0.018 & 0.647 & 0.025 & 0.748 & 0.025 & No & \\
\hline F3_0692 & 024057.994 & +424944.614 & & 17.136 & 0.010 & 0.857 & 0.016 & 1.061 & 0.015 & No & \\
\hline F3_2310 & 024044.085 & +425358.059 & & 99.999 & 9.999 & 9.999 & 9.999 & 9.999 & 9.999 & & \\
\hline F4_0167 & 024148.825 & +423131.702 & JP 223 & 13.502 & 0.021 & 0.938 & 0.026 & 1.030 & 0.031 & No & \\
\hline F4_0404 & 024127.663 & +423542.306 & JP 131 & 14.730 & 0.010 & 0.852 & 0.014 & 0.932 & 0.014 & Yes & No \\
\hline F4_0515 & 024127.188 & +424341.881 & JP 129 & 15.111 & 0.010 & 0.903 & 0.014 & 0.977 & 0.014 & No & \\
\hline
\end{tabular}

Notes. ${ }^{(a)}$ Identifier format is XX_YYYY, where "XX" are representations F3 and F4 for targets detected on M 34 western Field 3 or western Field 4 images, and "YYYY" are internal catalog numbers.

(b) Astrometric data are derived from coordinate matches in the SuperCosmos survey catalogs (Hambly et al. 2001a-c).

(c) JP 96 identifiers are for photometric variables which are correlated with Jones \& Prosser (1996) sources.

(d) BVI photometric data are taken from the Indiana Group survey (cf., Sect. 3).

(e) Membership assignments are based upon whether a target star lies on (or close to) the $V / B-V$ and/or $V / V-I$ main sequence loci (see Fig. 1) or whether a target star has a radial velocity consistent with the cluster's systemic velocity (Meibom et al., in prep.; see also Sect. 2.1 and Meibom et al. 2006).

(f) $B-V$ colours are determined using a field star $V-I$ to $B-V$ relationship (Caldwell et al. 1993), assuming $E_{V-I}=1.25 \times E_{B-V}$, and $E_{B-V}=$ 0.07 (Canterna et al. 1979). 
A\&A 515, A100 (2010)

Table 3. Photometric periods and ancillary data are presented, resulting from the light-curve analysis of variable stars detected in the Lowell M 34 fields.

\begin{tabular}{|c|c|c|c|c|c|}
\hline $\begin{array}{l}\text { Internal }^{a} \\
\text { Identifier }\end{array}$ & $\begin{array}{l}\text { Data }^{b} \\
\text { Epochs }\end{array}$ & $\begin{array}{l}\text { Sigma Clipped }^{c} \\
\text { StdDev [mag] }\end{array}$ & $\begin{array}{l}\text { Period } \\
\text { [days] }\end{array}$ & $B-V_{o}^{d}$ & $\mathrm{Mem}^{e}$ \\
\hline F3_0172-IIIVsV & $53 / 54 / 59$ & $0.00883 / 0.01010 / 0.01190$ & $8.0 \pm 1.0$ & 0.811 & $\mathrm{Y} ; \mathrm{Ys}$ \\
\hline F3_0176-IIIVsIsV & $53 / 54 / 58 / 59$ & $0.04275 / 0.06460 / 0.04313 / 0.06427$ & $2.780 \pm 0.005$ & 0.818 & Y;Ys \\
\hline F3_0215-IIIVsIsV & $52 / 54 / 58 / 59$ & $0.01121 / 0.01722 / 0.01439 / 0.02020$ & $7.47 \pm 0.11$ & 0.874 & $\mathrm{Y} ; \mathrm{Ys}$ \\
\hline F3_0258-1IIVsV & $53 / 54 / 58$ & $0.01182 / 0.01250 / 0.01457$ & $11.0 \pm 1.0$ & 0.947 & $\mathrm{Y} ; \mathrm{Ys}$ \\
\hline F3_0306-IIsIsV & $53 / 58 / 59$ & $0.01886 / 0.01809 / 0.02081$ & $1.1435 \pm 0.0007$ & 0.991 & $\mathrm{Y} ; \mathrm{Yb}$ \\
\hline F3_0320-1IIVsV & $52 / 54 / 58$ & $0.00898 / 0.01023 / 0.01430$ & $8.511 \pm 0.001$ & 1.046 & $\mathrm{Y}$ \\
\hline F3_0383-1IlV & $52 / 54$ & $0.00776 / 0.00937$ & $9.5 \pm 0.4$ & 1.160 & $\mathrm{Y} ; \mathrm{Ys}$ \\
\hline F3_0430-1V & 54 & 0.01253 & $10.5 \pm 1.0$ & 1.239 & $\mathrm{Y} ; \mathrm{Ys}$ \\
\hline F3_0469-IIIVsIsV & $52 / 54 / 57 / 57$ & $0.01344 / 0.01745 / 0.01768 / 0.02225$ & $12.9 \pm 0.3$ & 1.283 & $\mathrm{Y}$ \\
\hline F3_0485-1IIV & $52 / 54$ & $0.01040 / 0.01393$ & $12.0 \pm 1.0$ & 1.285 & Y; \\
\hline F3_0487-1IIV & $53 / 54$ & $0.01076 / 0.01388$ & $8.4 \pm 0.6$ & 1.278 & $\mathrm{Y}$ \\
\hline F3_0600-1IIV & $53 / 54$ & $0.01263 / 0.02503$ & $7.44 \pm 0.05$ & 1.452 & $\mathrm{Y}$ \\
\hline F3_0664-II & 52 & 0.01077 & $10.5 \pm 1.0$ & 1.441 & $\mathrm{Y}$ \\
\hline F4_0136-sIsV & $60 / 60$ & $0.01457 / 0.01794$ & $2.87 \pm 0.03$ & 0.544 & $\mathrm{Y} ; \mathrm{Ys}$ \\
\hline F4_0169-IIIVsIsV & $55 / 42 / 60 / 59$ & $0.01275 / 0.01297 / 0.01094 / 0.01429$ & $3.81 \pm 0.08$ & 0.598 & $\mathrm{Y} ; \mathrm{Ys}$ \\
\hline F4_0194-sIsV & $60 / 60$ & $0.00817 / 0.00889$ & $2.20 \pm 0.03$ & 0.652 & $\mathrm{Y} ; \mathrm{Ys}$ \\
\hline F4_0234-IIIVsV & $54 / 55 / 60$ & $0.00692 / 0.00984 / 0.01014$ & $5.56 \pm 0.07$ & 0.698 & $\mathrm{Y} ; \mathrm{Ys}$ \\
\hline F4_0303-IIIVsIsV & $55 / 55 / 60 / 60$ & $0.00800 / 0.00941 / 0.00839 / 0.00978$ & $7.4 \pm 0.3$ & 0.767 & $\mathrm{Y} ; \mathrm{Ys}$ \\
\hline F4_0317-IIIVsIsV & $55 / 55 / 59 / 60$ & $0.01701 / 0.02159 / 0.01698 / 0.02167$ & $6.8 \pm 0.1$ & 0.805 & $\mathrm{Y} ; \mathrm{Ys}$ \\
\hline F4_0327-1VsV & $55 / 60$ & $0.01471 / 0.01406$ & $7.6 \pm 1.1$ & 0.813 & $\mathrm{Y} ; \mathrm{Ys}$ \\
\hline F4_0335-IIIVsIsV & $55 / 55 / 60 / 60$ & $0.01538 / 0.01953 / 0.01601 / 0.02032$ & $0.89 \pm 0.02$ & 0.790 & $\mathrm{Y} ; \mathrm{Yb}$ \\
\hline F4_0450-IIIVsIsV & $54 / 55 / 60 / 60$ & $0.00985 / 0.01181 / 0.01264 / 0.01368$ & $8.00 \pm 0.21$ & 0.895 & Y;Ys \\
\hline F4_0667-IIIVsV & $54 / 55 / 59$ & $0.01122 / 0.01819 / 0.02028$ & $7.97 \pm 0.05$ & 1.030 & $\mathrm{Y} ; \mathrm{Yb}$ \\
\hline F4_0695-II & 54 & 0.00748 & $4.3 \pm 0.5$ & 1.086 & Y;Ys \\
\hline F4_0730-IIIVsIsV & $55 / 55 / 60 / 59$ & $0.02944 / 0.04619 / 0.03109 / 0.04695$ & $8.41 \pm 0.10$ & 1.051 & $\mathrm{Y}$ \\
\hline F4_0736-IIIVsIsV & $55 / 55 / 60 / 60$ & $0.01875 / 0.02715 / 0.02022 / 0.02911$ & $0.786 \pm 0.001$ & 1.008 & $\mathrm{Y} ; \mathrm{Yb}$ \\
\hline F4_0803-1VsI & $55 / 60$ & $0.01435 / 0.01416$ & $12.6 \pm 0.7$ & 1.164 & Y;Ys \\
\hline F4_0804-1IIVsIsV & $55 / 55 / 60 / 60$ & $0.02568 / 0.04152 / 0.02448 / 0.04239$ & $4.95 \pm 0.04$ & 1.106 & $\mathrm{Y}$ \\
\hline F4_0852-1IIVsIsV & $55 / 55 / 59 / 60$ & $0.01547 / 0.02368 / 0.01624 / 0.02564$ & $0.879 \pm 0.002$ & 1.155 & $\mathrm{Y} ; \mathrm{Yb}$ \\
\hline F4_0942-1IIVsV & $54 / 55 / 60$ & $0.00942 / 0.01300 / 0.02362$ & $9.4 \pm 0.3$ & 1.198 & $\mathrm{Y}$ \\
\hline F4_1007-IIIV & $55 / 55$ & $0.00774 / 0.01177$ & $15 \pm 2$ & 1.440 & $\mathrm{Y}$ \\
\hline F4_1020-IIIVsIsV & $55 / 55 / 60 / 59$ & $0.01344 / 0.01846 / 0.01568 / 0.02528$ & $8.6 \pm 0.4$ & 1.260 & $\mathrm{Y}$ \\
\hline F4_1055-IIIVsIsV & $55 / 55 / 60 / 60$ & $0.01427 / 0.02542 / 0.02233 / 0.02664$ & $6.80 \pm 0.15$ & 1.272 & $\mathrm{Y}$ \\
\hline F4_1147-IV & 55 & 0.01883 & $0.589 \pm 0.001$ & 1.336 & $\mathrm{Y}$ \\
\hline F4_1315-IIIV & $54 / 54$ & $0.01429 / 0.01913$ & $12.2 \pm 0.5$ & 1.371 & $\mathrm{Y}$ \\
\hline F4_1357-1IIV & $55 / 55$ & $0.01197 / 0.01973$ & $9.4 \pm 0.2$ & 1.380 & $\mathrm{Y}$ \\
\hline F4_1369-1IIV & $53 / 53$ & $0.01886 / 0.02578$ & $11.85 \pm 0.13$ & 1.443 & $\mathrm{Y}$ \\
\hline F4_1392-IIIV & $55 / 55$ & $0.01496 / 0.02385$ & $6.5 \pm 0.5$ & 1.434 & $\mathrm{Y}$ \\
\hline F4_1566-IIIV & $55 / 54$ & $0.01920 / 0.03248$ & $11.2 \pm 0.1$ & 1.479 & $\mathrm{Y}$ \\
\hline F4_1617-1V & 52 & 0.03379 & $7.3 \pm 0.5$ & 1.500 & $\mathrm{Y}$ \\
\hline F4_1793-IIIV & $55 / 53$ & $0.02221 / 0.04966$ & $7.5 \pm 0.6$ & 1.323 & $\mathrm{Y}$ \\
\hline F4_1925-1V ${ }^{f}$ & 44 & 0.09210 & $12.7 \pm 2.0$ & 1.507 & $\mathrm{Y}$ \\
\hline F4_2226-II ${ }^{f}$ & 55 & 0.02681 & $6.0 \pm 0.5$ & 1.555 & $\mathrm{Y}$ \\
\hline F3_0071-sIsV & $58 / 59$ & $0.02052 / 0.02502$ & $3.014 \pm 0.008$ & 0.977 & $\mathrm{~N}$ \\
\hline F3_0121-1VsV & $54 / 59$ & $0.00851 / 0.00881$ & $8.4 \pm 0.2$ & 1.178 & $\mathrm{~N}$ \\
\hline F3_0163-IIsIsV & $53 / 58 / 59$ & $0.00814 / 0.00943 / 0.00935$ & $14 \pm 2$ & 0.830 & $\mathrm{Y} ; \mathrm{N}$ \\
\hline F3_0165-II & 52 & 0.01089 & $12 \pm 1$ & 0.795 & $\mathrm{Y} ; \mathrm{N}$ \\
\hline F3_0388-IIIVsIsV & $53 / 54 / 58 / 59$ & $0.01534 / 0.02141 / 0.01715 / 0.02448$ & $11.59 \pm 0.01$ & 0.905 & $\mathrm{~N}$ \\
\hline F3_0413-IIIVsIsV & $53 / 54 / 58 / 59$ & $0.01640 / 0.02417 / 0.01909 / 0.02982$ & $5.45 \pm 0.08$ & 1.073 & $\mathrm{Y} ; \mathrm{N}$ \\
\hline F3_0437-1IIVsV & $53 / 54 / 59$ & $0.00859 / 0.01134 / 0.01563$ & $9.7 \pm 0.4$ & 0.577 & $\mathrm{~N}$ \\
\hline F3_0692-IIIV & $53 / 54$ & $0.01886 / 0.02686$ & $0.3368 \pm 0.0001$ & 0.787 & $\mathrm{~N}$ \\
\hline F3_2310-1IIV & $52 / 54$ & $0.02667 / 0.02846$ & $2.991 \pm 0.008$ & - - & \\
\hline F4_0167-IIIVsI & $54 / 55 / 59$ & $0.01321 / 0.01091 / 0.00961$ & $9.2 \pm 0.9$ & 0.868 & $\mathrm{~N}$ \\
\hline F4_0404-IIIVsV & $55 / 55 / 60$ & $0.01099 / 0.01419 / 0.01461$ & $6.6 \pm 0.3$ & 0.782 & $\mathrm{Y} ; \mathrm{N}$ \\
\hline F4_0515-1IlVsV & $55 / 55 / 60$ & $0.00886 / 0.00957 / 0.01239$ & $8.2 \pm 0.1$ & 0.833 & $\mathrm{~N}$ \\
\hline
\end{tabular}

Notes. ${ }^{(a)}$ Internal Identifiers are the same as those detailed in Table 2. Designations indicating exposure time and filter choice for a given lightcurve are annotated. For instance, II represents long-I, for light-curves derived from long exposures in the $I$-filter. Thus, IIIVsIsV represents four independent light-curves created from image sequences of $400 \mathrm{~s}, I$-filter; $600 \mathrm{~s}, V$-filter; $40 \mathrm{~s}, I$-filter and $60 \mathrm{~s}, V$-filter, respectively (namely, long-I, long-V, short-I \& short-V).

(b) Number of photometric data points in each light-curve, derived from each target's image series, in a given filter and exposure time sequence.

(c) For each target's light-curve, in a filter+exposure combination, standard deviations of the differential magnitudes are provided. The time series files for each target were sigma-clipped to eliminate outliers. A $\Delta$-magnitude for each light-curve can be determined by multiplying these standard deviations by $2 \sqrt{2}$ (strictly speaking this is only true for perfectly sinusoidal variations).

(d) $E_{B-V}=0.07$ is adopted; Canterna et al. (1979).

(e) Abridged cluster membership criteria are reproduced from Table 2, with format Photometric; Kinematic (s-single, b-binary).

(f) $B-V$ colours are determined using a field star $V-I$ to $B-V$ relationship (Caldwell et al. 1993), assuming $E_{V-I}=1.25 \times E_{B-V}$, and $E_{B-V}=0.07$. 

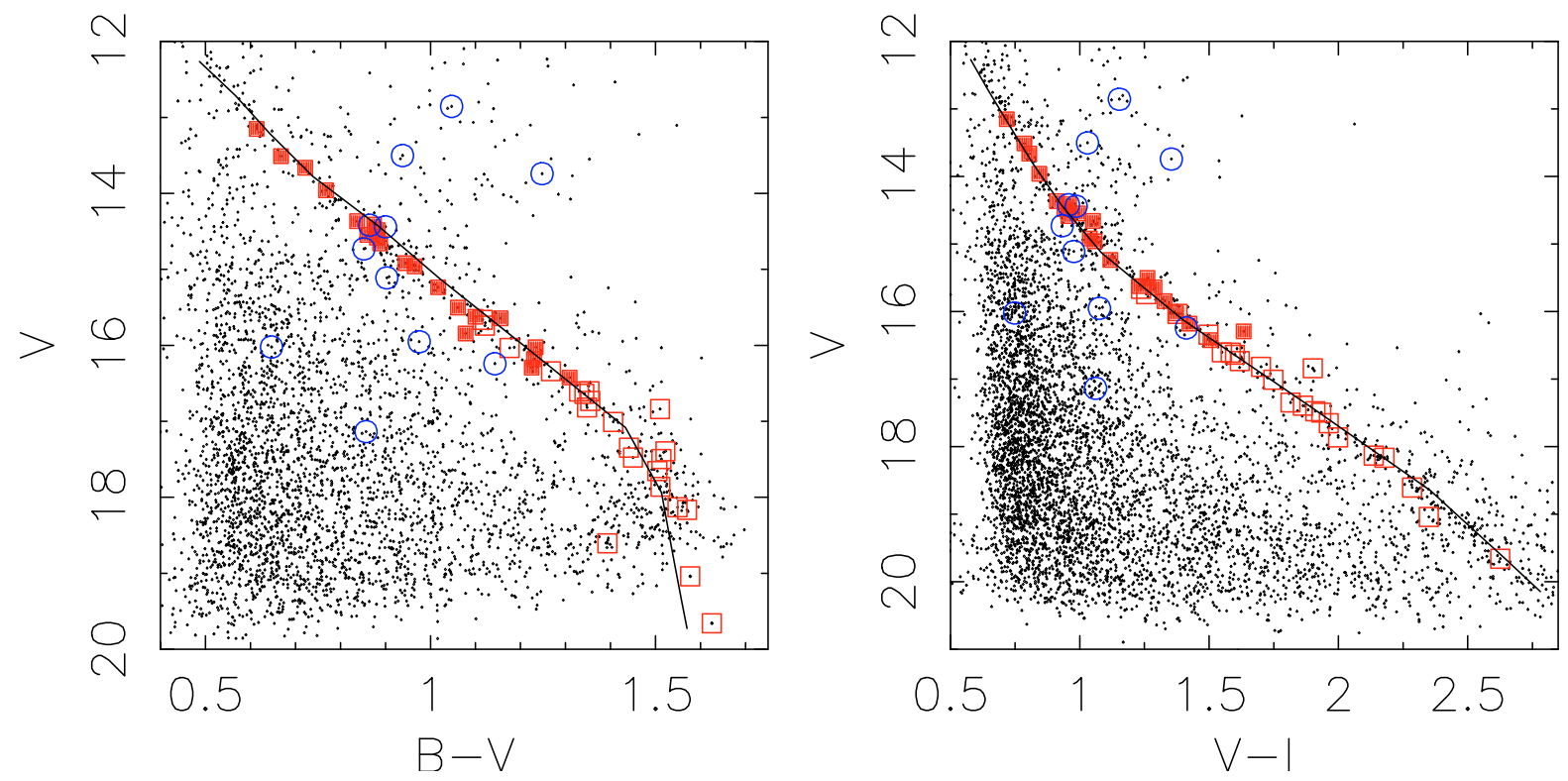

Fig. 1. Colour magnitude diagrams, in $B-V$ and $V-I$ colours, are presented for the entire photometry catalogue of the M $34 B V I c$ survey conducted by the Indiana Group. Solid lines represent D'Antona \& Mazzitelli (1997) theoretical isochrones, for a cluster age of $250 \mathrm{Myr}$, a distance of $470 \mathrm{pc}$, and assuming $E_{V-I}=1.25 \times E_{B-V}$, and $E_{B-V}=0.07$ (Canterna et al. 1979). Red squares, highlighting photometric and radial velocity members of the cluster (solid symbols), and photometric cluster membership only (open symbols) are plotted for M 34 stars for which we have derived photometric periods during the Lowell campaign. Blue circles depict those other periodic photometric variables in the field of M 34 which are photometric and/or radial velocity non-members of the cluster.

time-series. Periods longer than our 13-day comfort timescale abut the sensitivity limit of our observing window, which result in consequentially larger period errors for the $[2 / 55]$ systems for which we report periods $>13$ days (see Table 3 ).

\subsection{Establishing membership of $M 34$ from photometric variability alone}

Constructing a colour-magnitude diagram is the simplest way of establishing possible membership of an open cluster because stars located far from the cluster sequence are guaranteed to be non-members. Stars on the cluster sequence may or may not be bona fide cluster members, depending on the extent of fore- and background contamination from the Galactic field. For stars forming an apparent cluster main sequence, additional information is needed to distinguish between true members and field-star, non-member interlopers. Classical, well established methods for member, non-member triage involve astrometric (proper motion) measurements or measuring radial velocities (e.g., Stauffer 1984; Meynet et al. 1993; Perryman et al. 1998; Raboud \& Mermilliod 1998; Mermilliod et al. 2008), however such measurements are not always practical to acquire. In their absence, magnetic activity (e.g., X-rays) or lithium abundance indicators can be used to distinguish between cluster and non-cluster stars (e.g., Prosser et al. 1995a; James \& Jeffries 1997; Cargile et al. 2009). Here, we propose that photometric periodicity can also be exploited to easily establish open cluster membership in a manner unbiased with respect to brightness, mass (at least for solar-type stars) and distance.

In Fig. 1, we plot colour magnitude diagrams of the cluster in $B V$ and $V I$ colours, along with D'Antona \& Mazzitelli (1997) theoretical isochrones for $250 \mathrm{Myr}$ and $470 \mathrm{pc}$ in both colours. The 55 periodic variables in our Lowell fields are also identified on the figure. Photometric and/or radial velocity members, 43 in number, are flagged with red squares, whereas non-members,
12 in number, are flagged with blue circles (their periodicity and other information are tabulated in Tables 2 and 3). The cluster sequence is traced out very well by the periodic variables. In fact, in this particular case, photometric periodicity information alone provides a 78\% (43/55) probability of cluster membership. We believe the reason for this behavior is that the cluster stars are younger and hence far more active and variable than the mostly older disk stars in the field. One might even envision using photometric variability to trace out cluster sequences in sparsely populated young clusters or background-dominated cluster fields.

\subsection{Comparison with prior periods}

Irwin et al. (2006) have published a prior study of periodic variables in M34, and before proceeding further, we compare our periods with theirs for stars in common between the two studies. This comparison is graphically represented in Fig. 2, where symbols are assigned to stars having a varying number of independent period derivations (1-4) in our dataset. Of the 19 stars in common between the Irwin et al. sample and our own Lowell campaign, roughly half $(9 / 19)$ have periods that agree within 10\%. For all but two of the remaining 10 stars, Irwin et al. periods are significantly shorter than ours, where the origins for such discrepancies are not entirely clear to us.

Through comparison with an as yet unpublished photometric variability study of M34 we can establish confidence in the Lowell campaign periods, and advocate their preferential use compared to the Irwin et al. ones. Three stars in common to the Irwin et al. study and our Lowell campaign, F3_0413, F4_0234, and F4_1147, have Meibom et al. (in prep.) periods, whose photometric variability was characterized over several months worth of observation data (e.g., see Meibom et al. 2009, for details of a similar observing programme for the M 35 cluster). These data, flagged stars in Fig. 2 and listed in Table 4, show that the 


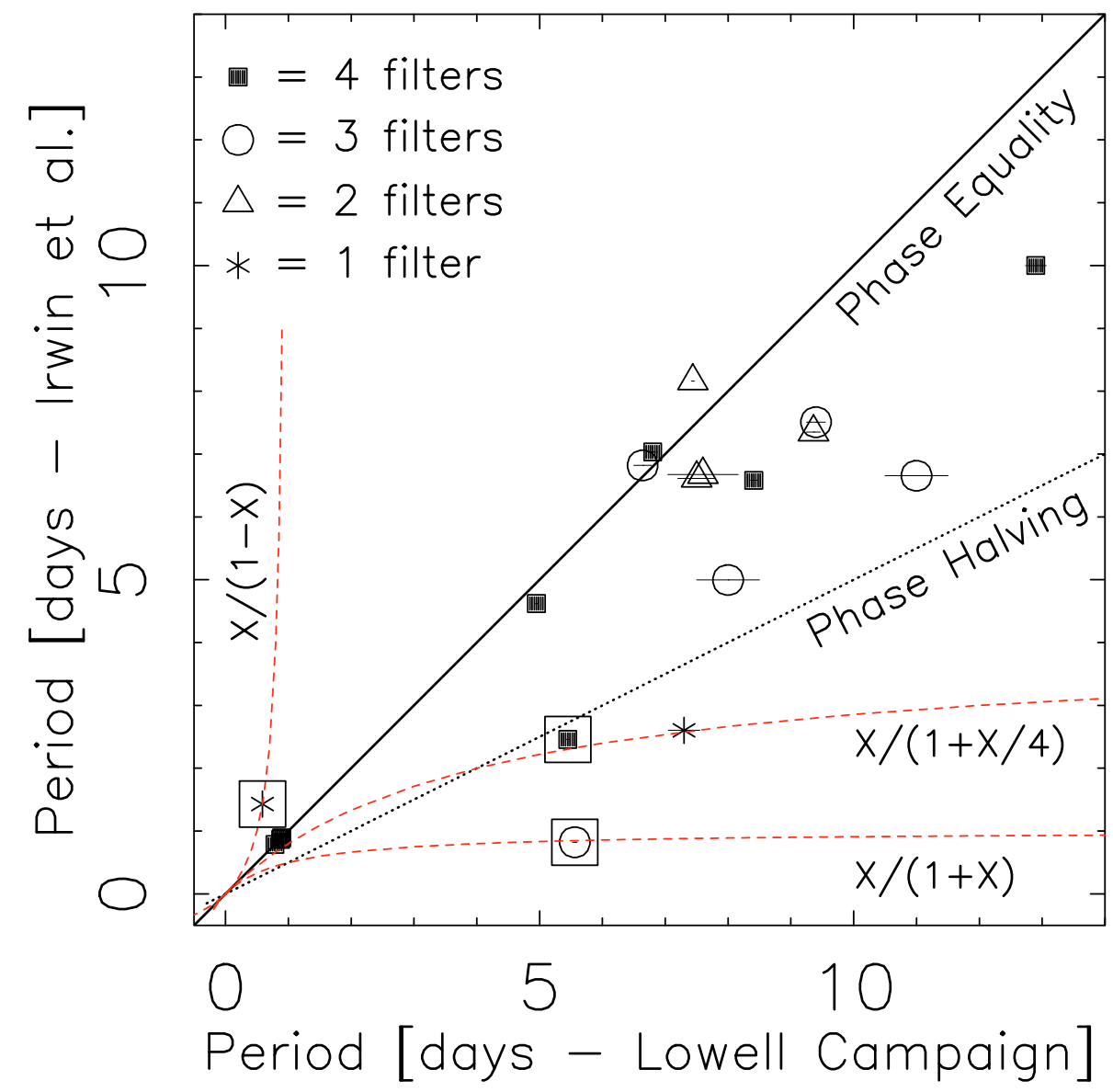

Fig. 2. Photometric periods for M 34 stars common to our Lowell campaign and the Irwin et al. study are shown. Periods determined from the Lowell campaign, using photometric lightcurves in up to four (4) different filter/exposure time combinations, are depicted by the filledsquare, circle, triangle and asterisk symbols (see Tables 2 and 3). We include error bars on our Lowell periods, which for many targets are smaller than the symbol sizes. No error bars are available for the Irwin et al. periods. The solid line represents equality between the two studies, and is not a fit to the data. The dotted line represents phase-halving while the dashed lines represent several period-aliasing loci, respectively. About one-third of the sample have equal period determinations (within the errors). Of the remainder, all but two of the Irwin et al. periods are shorter than ours. See text for possible reasons for the discrepancy (see Sect. 4.4). The three flagged stars (with boxed symbols) are discrepant ones where our periods have been confirmed by a forthcoming Meibom et al. (in prep.) study.
Table 4. A comparison of photometric periods for three M34 stars common to the Lowell campaign, the Irwin et al. study and the Meibom et al. (in prep.) study (cf., Fig. 2 and Sect. 4.4).

\begin{tabular}{cccc}
\hline \hline ID & $\begin{array}{c}\text { Lowell } \\
\text { [days] }\end{array}$ & $\begin{array}{c}\text { Irwin et al. } \\
\text { [days] }\end{array}$ & $\begin{array}{c}\text { Meibom et al. } \\
\text { [days] }\end{array}$ \\
\hline F3_0413 & $5.45 \pm 0.08$ & 2.458 & 5.47 \\
F4_0234 & $5.56 \pm 0.07$ & 0.822 & 5.47 \\
F4_1147 & $0.589 \pm 0.001$ & 1.429 & 0.592 \\
\hline
\end{tabular}

Meibom et al. periods are an excellent match to the Lowell periods (to within 1\%), whereas the Irwin et al. periods are poorly matched.

Proving the correctness of one period derivation over another is not an easy task. However, we note that our dataset has a 17-night baseline compared with the 10-night baseline of Irwin et al., suggesting that we have better sensitivity to longer period systems. Our data were also acquired all night, rather than restricted to half nights as the Irwin et al. observational data were. These facts strongly suggest that our data are less sensitive to period aliasing; indeed, several of the Irwin et al. stars lie on or close to particular alias curves, as shown in Fig. 2. Finally, we note that most of our periods [17/19] are confirmed in at least a second filter/exposure time combination in the Lowell campaign data. Consequently, in light of the concerns that we have for aliasing effects in some of the Irwin et al. data, we will preferentially use our own periods for the remainder of this work.

\section{The photometric period distribution in M 34}

Our rotation period distribution for photometric and/or kinematic members of M34, as a function of intrinsic $B-V$ and $V-I$ colours, is displayed in Fig. 3. In agreement with prior results in various open clusters (B03, B07), there are no stars located in the upper left portion of the colour-period diagrams. The Sun-like $\left[(B-V)_{o} \simeq 0.64\right]$ stars in our M 34 sample are rotating about an order of magnitude faster than the Sun. However the lower mass stars, mid-K and later, have an order-of-magnitude spread in rotation period, as has been seen before in other young open clusters. Further detailed interpretation of the M 34 colourperiod diagram requires a comparison of the colour-period distributions for other young clusters.

Rotation period distributions for two comparison clusters whose ages straddle that of M34 will allow us to critically investigate the gyrochronological properties of M 34. To this end, we display colour-period diagrams for the $\simeq 135 \mathrm{Myr}$ M 35 and 600 Myr Hyades clusters in Fig. 4. Colour-period data are taken from the recent study of M 35 by Meibom et al. (2009), and for the Hyades, from Radick et al. (1987) and Prosser et al. (1995b). This side-by-side comparison shows that the $\sim 135$ Myr-old M 35 cluster has two distinct sequences of rotating stars indicated by the solid and dashed line in the figure, while the older Hyades cluster has only one. B03 called these sequences "I" (for Interface) and "C" (for Convective), with "g" (for gap) stars in transition from $\mathrm{C}$ to I (see Sect. 1 for a discussion of the angular momentum characteristics of $\mathrm{CgI}$ stars). What we see plainly from the comparison is that by the Hyades age, most rotating solar-type stars have been transformed into I-type stars. Graphs of this C-g-I transformation for a series of clusters can be found in B03 and Meibom et al. (2009). 
D. J. James et al.: The gyrochronology age of M34
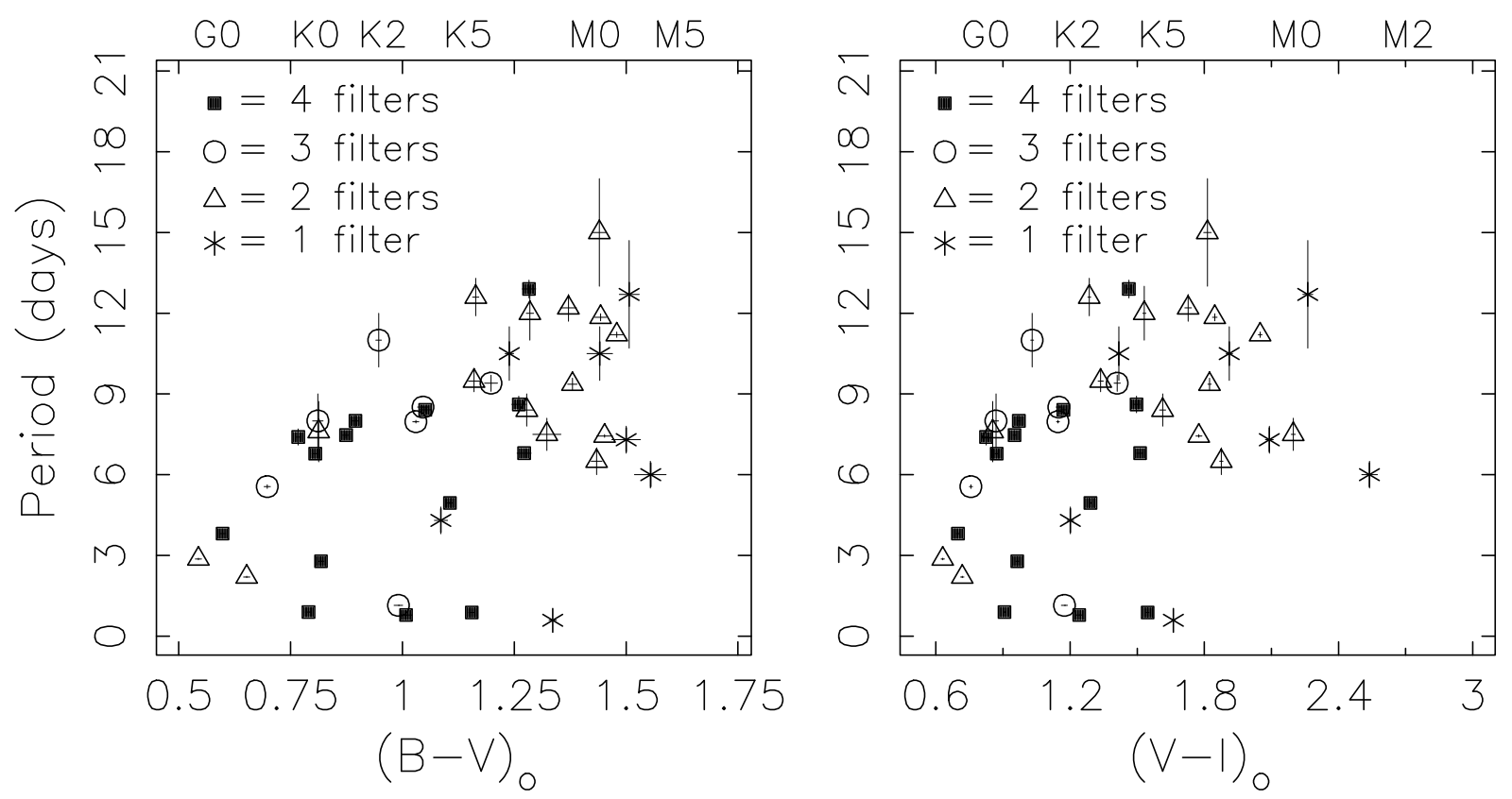

Fig. 3. Photometric period distributions, as a function of intrinsic colours $(B-V)_{o}$ and $(V-I)_{o}$, are plotted for solar-type stars in NGC 1039 (M34). We adopt $E_{B-V}=0.07$ (Canterna et al. 1979), and $E_{V-I}=1.25 \times E_{B-V}$. Period symbols are the same as those depicted in Fig. 2. Error bars are included, and are for some targets smaller than the symbol sizes. Approximate spectral types for a given colour are annotated on the upper abscissae (from Zombeck 1990).
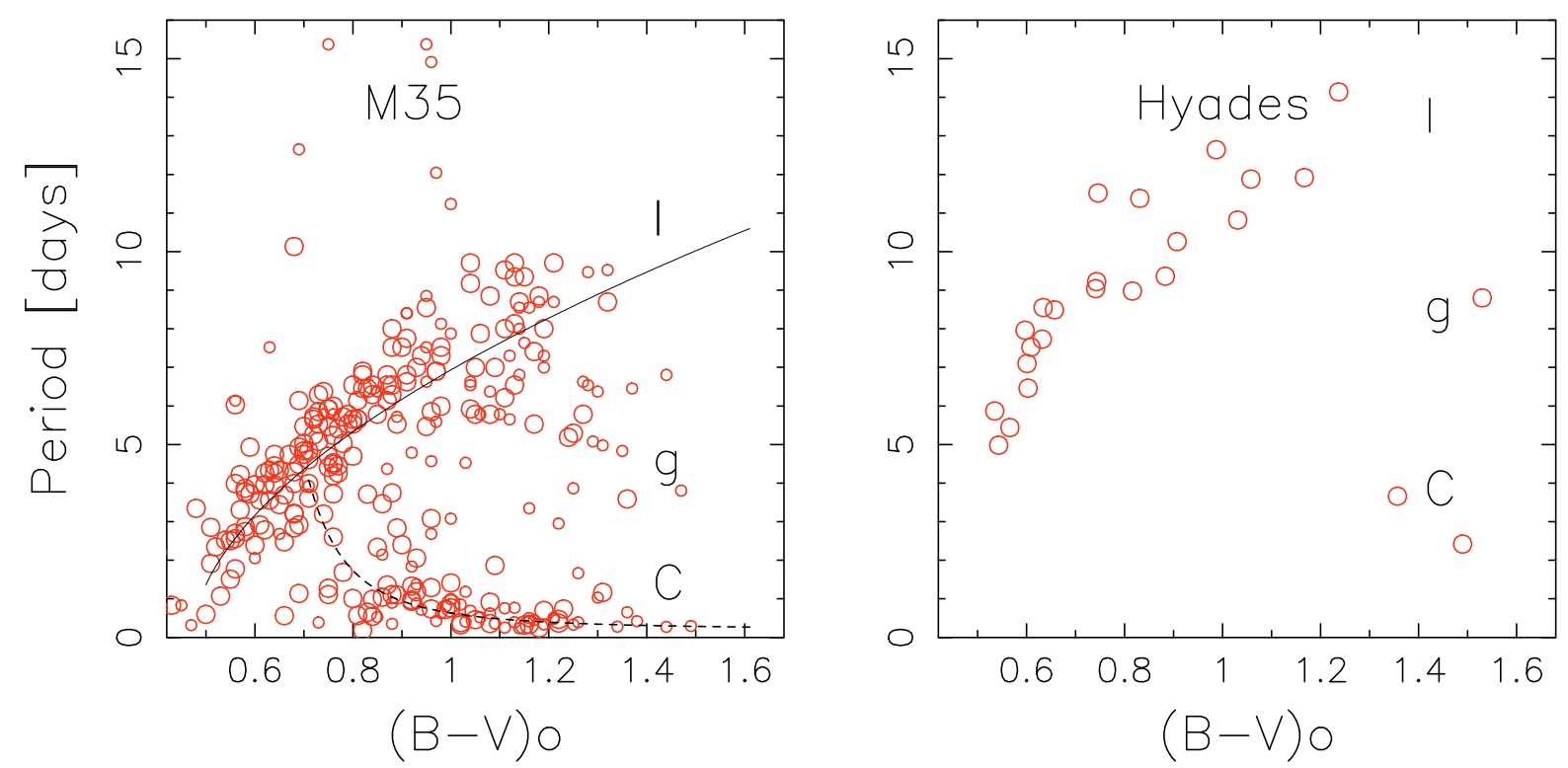

Fig. 4. In the left-hand panel, the rotation period distribution (in days) of the $\simeq 135 \mathrm{Myr}$ M 35 cluster is plotted against intrinsic $(B-V$ ) colour (data from Meibom et al. 2009); stars which are both photometric and kinematic members of the cluster are plotted with larger symbols than stars having photometric membership alone. Over-plotted are $135 \mathrm{Myr}$ interface [solid line] and convection [dashed line] gyrochronology loci from the calibration by Meibom et al. (2009). In the right-hand panel, similar colour-period data are plotted for the 600 Myr Hyades cluster (data from Radick et al. 1987; Prosser et al. 1995b). Under the paradigm of gyrochronology, specific regions of the colour-period distributions are identified with C [convective], g [gap] and I [interface] symbols.

We now take these gyrochronology sequences from the M 35 colour-period distribution, and overplot them on the M34 data in the left hand panel of Fig. 5. This allows us to classify the gyrochronology status of the (far fewer) M 34 stars roughly, as indicated by the $\mathrm{CgI}$ symbols in the figure. We note that its $\mathrm{C}$ sequence is in the same approximate position as that of M 35 , but its I sequence lies above that of M 35, as expected from its older age. The manner of this inter-comparison suggests that only the stars shown in the right panel of the figure ought to be identified as I-sequence stars in M 34, which we henceforth do.

\subsection{A gyrochronology age for $M 34$}

It is desirable to derive an age for M34 using our photometric period dataset, and the method of gyrochronology (B03, B07) 

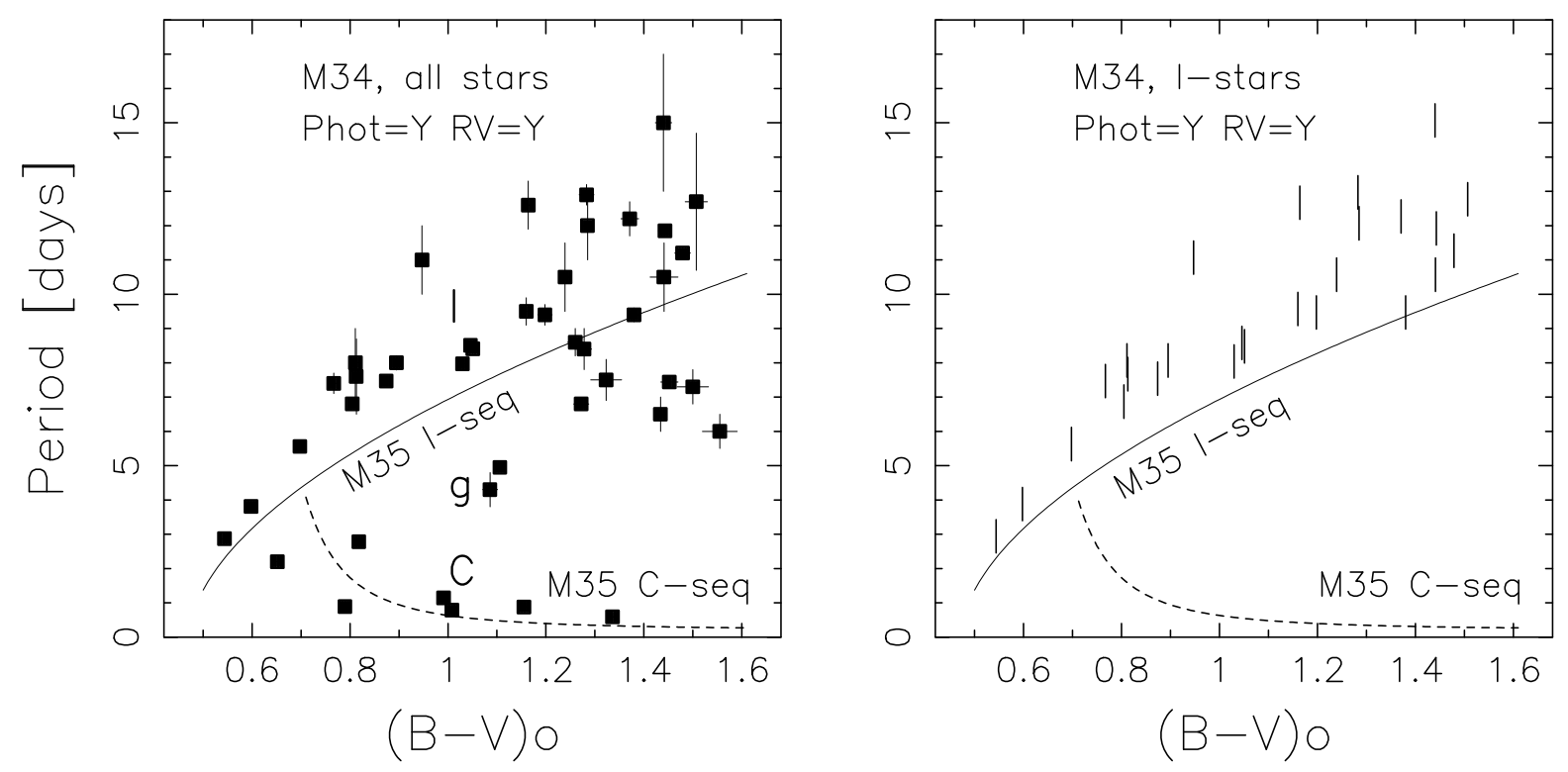

Fig. 5. The colour-period distribution for all photometric and/or kinematic members of M 34 (see Table 3) is plotted in the left hand panel. Overplotted are gyrochronology Interface and Convection sequence loci (solid \& dashed lines respectively) for the 135 Myr M 35 cluster (Barnes 2003 ; Meibom et al. 2009). Regions of the colour-period diagram where approximately $200 \mathrm{Myr}$ stars, classified as Interface, gap and Convective status in gyrochronology space, are identified with I, g or C symbols respectively. Candidate Interface sequence stars in the M 34 cluster, lying above the Meibom et al. M 35 Interface sequence, are identified as I-symbols in the right-hand panel; see Sect. 5 for details.

will allow us to pursue such an analysis. This exercise will allow us to examine whether the gyro age derived is physically reasonable, and hence whether it might be usable for other clusters in future. The basis for such a gyro age calculation relies upon;

1. deriving the true rotation period distribution of the cluster;

2. removing possible cluster non-members; and

3. choosing, and using only, the I-sequence stars in the cluster for the analysis.

For M34, we have performed these three steps to the best of our ability, as described above, with the resulting set of candidate I-sequence stars in the cluster being displayed in the righthand panel of Fig. 5. We note that the forthcoming study by Meibom et al. (in prep.), which contains many more M34 stars, will make the $\mathrm{CgI}$ distinction in this cluster more obvious, and verify our classifications made here. Based on fitting the open cluster colour-period distributions then available, B07 suggested that the positions of the I sequences of open clusters, or indeed that of field stars, follows the relationship;

$P_{\text {rot }}=f(B-V) \cdot g(t)$

where $P_{\text {rot }},(B-V)$, and $t$ represent the measured rotation period, measured de-reddened colour, and the age respectively, and $f$ and $g$ are fitted functions of the stellar colour and age respectively, where he proposed that

$f(B-V)=a(B-V-c)^{b}$

and

$g(t)=t^{n}$

for which Barnes (2007) derived coefficients of $a=0.7725 \pm$ $0.0110, b=0.601 \pm 0.024, c=0.4$, and $n=0.5189 \pm 0.0070$. However, based on the most extensive open cluster rotation period study to date, that of M 35 by Meibom et al. (2009 - displayed in the left-hand panel of Fig. 4), the coefficients of the
I-sequence fit have been recently updated to be $a=0.770 \pm$ $0.014, b=0.553 \pm 0.052$, and $c=0.472 \pm 0.027$ with a fixed $n=0.52$. However, the index $n$ is set by the solar calibration, by demanding that the gyrochronology relationship for the I-sequence yields the solar rotation period at the solar age, the rationale for which is explained at length in B07. We note that, using their extensive rotation period dataset for M 35, Meibom et al. re-fit the colour function coefficients of Eq. (2) but did not re-fit the age exponent, $n$, of Eq. (3). Therefore to be mathematically rigorous, we employ the Meibom et al. (2009) $a-c$ coefficients and the solar data summarized in Barnes (2007) to rederive the $g(t)$ exponent to be $n=0.5344 \pm 0.0015$.

Taking the Meibom et al. (2009) $a-c$ coefficients and the newly derived $n$ exponent, we fit the Eq. (1) relationship to the colour-period data of M34 I-sequence stars, those identified in Fig. 5, in order to determine the cluster's gyro age, the one free parameter in the fit. The results of this procedure are reported in Table 5. An unweighted fit to these data yields a rotation age for M 34 to be $193 \pm 9 \mathrm{Myr}^{3}$ ( \pm possible systematic errors in gyrochronology), where the quoted error is the error on the mean for these data. Such a gyro age for the cluster is consistent with the Jones \& Prosser and Meynet et al. isochronal ages for M 34.

\subsection{Variance of the derived periods about the fit}

Let us see if we can clarify the origin of items in the error budget contributing to the uncertainty in the cluster's gyrochronology age, which are in addition to any systematic error of the gyrochronology method itself. We can estimate the magnitude of the error budget by considering the additive contribution

${ }^{3}$ Using all of the Meibom et al. (2009) coefficients, including $n=$ 0.52 , a resultant gyrochronology age for M 34 of $223 \pm 11$ Myr would be obtained. 
Table 5. Gyrochronological ages for I-sequence stars in M34.

\begin{tabular}{llccllll}
\hline \hline Identifier & $\begin{array}{c}\text { JP }^{a} \\
\text { No. }\end{array}$ & $\begin{array}{c}\text { \# of } \\
\text { periods } \\
\text { derived }^{b}\end{array}$ & $\begin{array}{c}(B-V)_{o} \\
\text { Feasured } \\
\text { Period } \\
\text { [days] }\end{array}$ & $\begin{array}{c}\text { Gyro Age }^{c} \\
{[\mathrm{Myr}]}\end{array}$ & $\begin{array}{c}\text { Fitted }^{d} \\
\text { Period } \\
\text { [days] }\end{array}$ & $\begin{array}{l}\text { Period }^{e} \\
\text { Variance }^{\text {[days }}{ }^{2}\end{array}$ \\
\hline F4_0136 & JP 213 & 2 & 0.544 & $2.87 \pm 0.03$ & $178.5 \pm 3.5$ & 2.99 & 0.0144 \\
F4_0169 & JP 50 & 4 & 0.598 & $3.81 \pm 0.08$ & $170.0 \pm 6.7$ & 4.08 & 0.0729 \\
F4_0234 & JP 148 & 3 & 0.698 & $5.56 \pm 0.07$ & $188.4 \pm 4.4$ & 5.63 & 0.0049 \\
F4_0303 & JP 227 & 4 & 0.767 & $7.4 \pm 0.3$ & $244.1 \pm 18.6$ & 6.5 & 0.81 \\
F4_0317 & JP 224 & 4 & 0.805 & $6.8 \pm 0.1$ & $183.8 \pm 5.0$ & 7.0 & 0.04 \\
F4_0327 & JP 199 & 2 & 0.813 & $7.6 \pm 1.1$ & $220.9 \pm 59.8$ & 7.1 & 0.25 \\
F3_0172 & JP 49 & 3 & 0.811 & $8.0 \pm 1.0$ & $244.6 \pm 57.2$ & 7.0 & 1.00 \\
F3_0215 & JP 41 & 4 & 0.874 & $7.47 \pm 0.11$ & $180.4 \pm 5.0$ & 7.74 & 0.0729 \\
F4_0450 & JP 113 & 4 & 0.895 & $8.00 \pm 0.21$ & $194.5 \pm 9.6$ & 7.96 & 0.0016 \\
F3_0258 & JP 289 & 3 & 0.947 & $11.0 \pm 1.0$ & $313.1 \pm 53.3$ & 8.5 & 6.25 \\
F4_0667 & JP 52 & 3 & 1.030 & $7.97 \pm 0.05$ & $145.0 \pm 1.7$ & 9.28 & 1.7161 \\
F3_0320 & JP 172 & 3 & 1.046 & $8.511 \pm 0.001$ & $159.3 \pm 0.1$ & 9.429 & 0.84272 \\
F4_0730 & JP 18 & 4 & 1.051 & $8.41 \pm 0.10$ & $154.4 \pm 3.4$ & 9.47 & 1.1236 \\
F3_0383 & & 2 & 1.160 & $9.5 \pm 0.4$ & $162.2 \pm 12.8$ & 10.4 & 0.81 \\
F4_0803 & JP 229 & 2 & 1.164 & $12.6 \pm 0.7$ & $273.5 \pm 28.5$ & 10.5 & 4.41 \\
F4_0942 & & 3 & 1.198 & $9.4 \pm 0.3$ & $150.4 \pm 9.0$ & 10.7 & 1.69 \\
F3_0430 & & 1 & 1.239 & $10.5 \pm 1.0$ & $174.8 \pm 31.2$ & 11.1 & 0.36 \\
F3_0485 & & 2 & 1.285 & $12.0 \pm 1.0$ & $211.3 \pm 33.0$ & 11.4 & 0.36 \\
F3_0469 & & 4 & 1.283 & $12.9 \pm 0.3$ & $242.5 \pm 10.6$ & 11.4 & 2.25 \\
F4_1315 & & 2 & 1.371 & $12.2 \pm 0.5$ & $196.4 \pm 15.1$ & 12.1 & 0.01 \\
F4_1357 & & 2 & 1.380 & $9.4 \pm 0.2$ & $119.3 \pm 4.8$ & 12.2 & 7.84 \\
F3_0664 & & 1 & 1.441 & $10.5 \pm 1.0$ & $137.2 \pm 24.5$ & 12.6 & 4.41 \\
F4_1369 & & 2 & 1.443 & $11.85 \pm 0.13$ & $171.7 \pm 3.5$ & 12.61 & 0.5776 \\
F4_1566 & & 2 & 1.479 & $11.2 \pm 0.1$ & $148.8 \pm 2.5$ & 12.9 & 2.89 \\
F4_1007 & & 2 & 1.440 & $15.0 \pm 2.0$ & $267.8 \pm 66.8$ & 12.6 & 5.76 \\
F4_1925 & & 1 & 1.507 & $12.7 \pm 2.0$ & $183.0 \pm 53.9$ & 13.1 & 0.16 \\
\hline
\end{tabular}

Notes. ${ }^{(a)} J P$ identifier from Jones \& Prosser (1996).

(b) Number of filter/exposure combinations yielding photometric period derivations for a given star.

(c) Gyro ages determined using Meibom et al. (2009) I-sequence colour function coefficients, viz $a=0.770 \pm 0.014, b=0.553 \pm 0.052$ and $c=0.472 \pm 0.027$, and an age function exponent $n=0.5344 \pm 0.0015$ (see Sect. 5.1).

(d) Assuming a mean gyrochronology age for M34 of 193 Myr (the mean of the ages calculated in Col. 6 - see Sect. 5.1) and the Meibom et al. (2009) coefficients, with $n=0.5344 \pm 0.0015$, we use intrinsic $(B-V)$ colours and Eq. (1) to derive a fitted period for each M 34 I-sequence star. (e) Each star's contribution to the period variance [(Measured Period - Fitted Period $)^{2}$ ] is noted, where the total variance is 43.73 days ${ }^{2}$, with a mean of 1.68 days $^{2}$.

of each star's period variance ${ }^{4}$. We might expect contributions to the period variance from cluster non-members, tidallyinteracting binaries within the cluster, a possible age spread within the cluster, period errors, differential rotation, and finally, one from initial variations in the cluster's natal angular momentum distribution. The data listed in Table 5 show that the total period variance about the fit is $\sim 43.7$ days $^{2}$.

It is beyond the scope of this paper to consider possible systematic errors in the gyrochronology method, which is calibrated using both the Sun and a selection of young open clusters with measured photometric periods. An understanding of this error will probably require another decade of rotational work on open clusters. It would be unsurprising if such a study eventually identified a 15-20\% systematic error in the application of gyrochronology to real colour-period samples.

The cluster colour-magnitude diagrams (see Fig. 1) suggest that 3 periodic field variables could have sneaked into our 43 member star sample had we not had the radial velocity measurements to reject them. This suggests that of our 22 purely

\footnotetext{
4 We define each star's period variance as (Measured Period - Fitted Period $)^{2}$, where the measured period is the photometric variability period determined from our differential photometry and the fitted period is that derived from Eq. (1), using dereddened $B-V$ colours, an assumed mean gyro age of $193 \mathrm{Myr}$ and the Meibom et al. (2009) $a-c$ coefficients, with an $n$ exponent, $n=0.5344 \pm 0.0015$.
}

photometrically chosen members (see Table 3), one possibly two are non-members, making this unlikely to be any significant contributor to the error. The mean variance contributor of any given star in our M 34 period sample is 1.68 days $^{2}$ (see Table 5), which means that non-member variance contribution from photometric members is at most $\simeq 3.4$ days $^{2}(\sim 8 \%$ of the variance total). Of the 26 candidate I-sequence stars in M 34, twelve of them are classified as photometric-only members of the cluster, i.e., having no kinematic classification (with a mean period variance of 2.33 days $^{2}$ ). Of these twelve, only three have noticeably large period variances (F4_1357, 7.84 days $^{2}$; F4_1007, 5.76 days $^{2}$; F3_0664, 4.41 days $\left.^{2}\right)$. In any case contribution to period variance, and hence gyro age errors, by cluster non-members is at a low level.

There is also a possibility that some scatter in the gyro ages for individual M34 stars is contributed by systems in tidally locked binaries, which will be addressed in greater detail in the Meibom et al. (in prep.) study. We note that of the candidate I-sequence stars reported in Table 5, 14/24 have kinematic classifications. Only one of these stars is a binary system, with no data being available as to whether it is tidally-locked or not. On average, one such data point would contribute 1.68 days $^{2}$ to the total variance, or $\sim 4 \%$.

Observational studies of other young open clusters and associations suggest that they are cöeval to within about a few Myr 

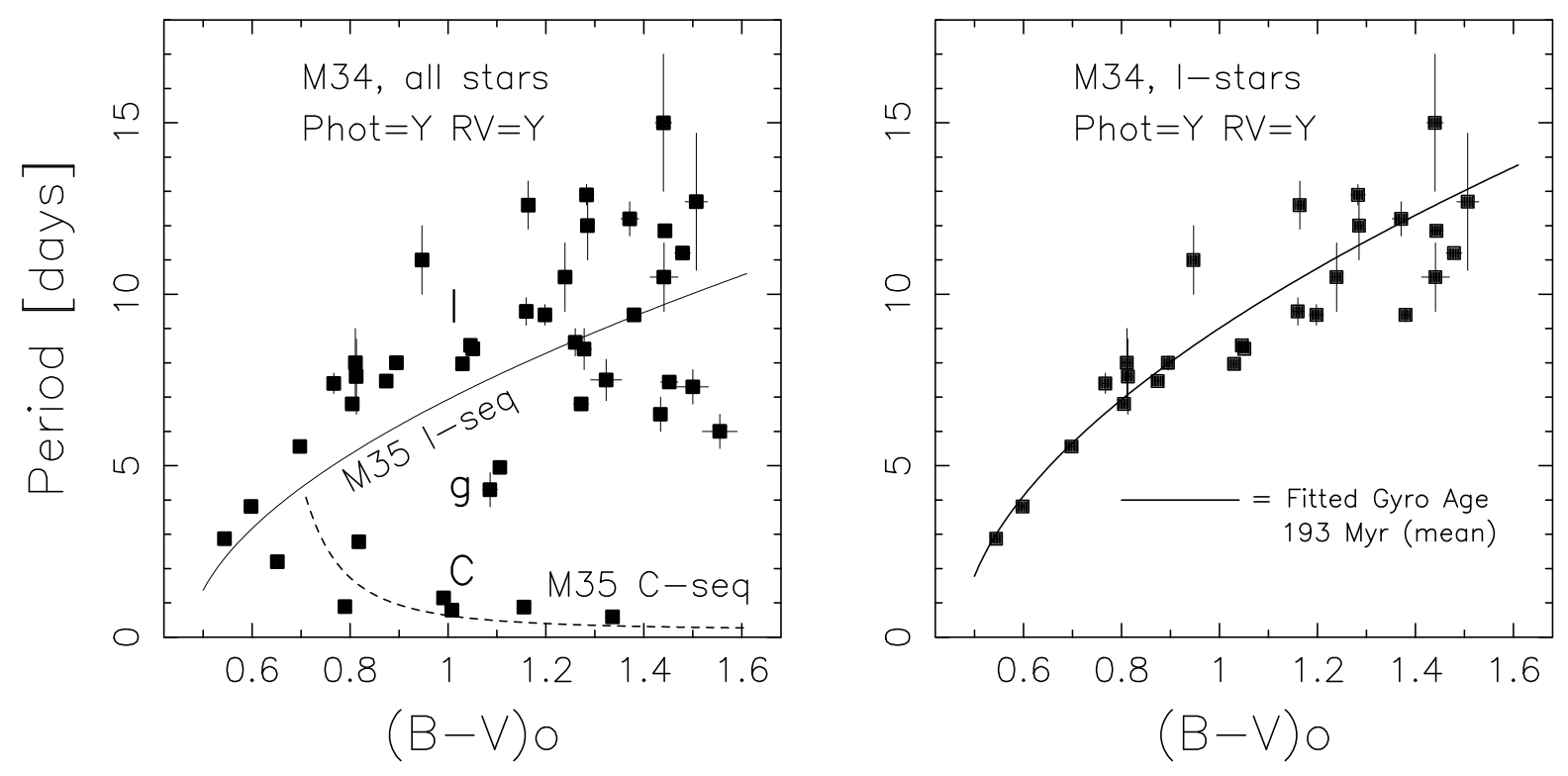

Fig. 6. This figure, very similar to Fig. 5, shows the colour-period data for our M 34 candidate I-sequence stars. The additional solid line in the right-hand panel represents the mean gyrochronology age of the I-stars in M 34 (193 Myr - see Table 5). This new gyrochronology I-sequence is calculated using updated colour-function coefficients (see Eqs. (1)-(3) and Meibom et al. 2009) together with our new derivation of the $g(t)$ exponent $(n=0.5344-$ see Eq. (3)).

(e.g., Wichmann et al. 2000; Palla \& Randich 2005; James et al. 2006). While spreads in observed quantities that also change with age are generally believed to originate in other ways, we note that recent theoretical work by Baraffe et al. (2009) shows that as a phenomenon, episodic accretion onto young solar-type stars can also act to introduce a luminosity spread, and hence age spread, in the main sequence loci of Hertzsprung Russell diagrams. An intrinsic age spread of 10 Myr (e.g., Jeffries 2009) at $B-V_{o}$ colours of $0.60,1.00$ and 1.40 in our $193 \mathrm{Myr}$ gyrochronology fit yields a maximum period variance of only 0.1 days $^{2}$. Such an intrinsic internal age spread within the cluster will therefore not contribute any significant variance.

Inspection of Fig. 5 and Table 3 shows that photometric period errors are quite large for the longer periods in our sample. We attribute this effect to the 17-night baseline of observations and expect that these errors will decline with a longer baseline dataset, such as the forthcoming Meibom et al. (in prep.) sample. Indeed, an informal comparison shows that they will be able to define the cluster's I-sequence better than our dataset allows. Another indication of this is that M34 I-sequence stars with smaller periods errors are, in general, located closer to the fitted sequence (see Fig. 6). Based solely on the period measurement errors themselves (see Table 5), the variance contribution due to imprecise period measurement is $\simeq 15.5$ days $^{2}(35.5 \%$ of the total).

Differential rotation must be present in this sample, in the sense that two stars with the same equatorial velocity might present spot groups at observed at different latitudes, introducing some period scatter in the observations. We do not however expect this to contribute much to the observed variance, based on the parametrization of its contribution to the fractional period variance via $\delta P / P=10^{-1.85} P^{0.3}$, following the analysis in Donahue et al. (1996) and Barnes (2007). For the I-sequence stars listed in Table 5, this effect contributes only something like $\simeq 2.06$ days $^{2}$ to the variance, equivalent to $5 \%$ of the total variance budget.
This leaves a remainder variance of 21.1 days $^{2}$ (48\% of the total) to be accounted for by the effects of either; (a) the systematic difference between the gyro fit and the observation data; or (b) the residual influence of initial variations. Because these two contributors have differing dependencies on cluster and observational parameters, it should be possible to understand their separate contributions as the rotation period database of open cluster stars expands.

\section{Discussion and summary}

We have presented the results of a 17-night differential photometry campaign over the Western half of the central region of the open cluster M 34. For all photometrically variable stars, we construct differential photometry light-curves, from which we derive periodicity. It is assumed that the photometric variability of objects consistent with cluster membership is due to the presence of magnetic-field induced starspots on their surfaces, rotating with the star; the derived periods are therefore representative of the angular velocity of the star.

In order to assess cluster membership, we exploit an extensive standardized CCD survey of the cluster, which shows that the majority of photometric variable stars [47/55] lie along the loci of the cluster main sequence in $V / B-V$ and $V / V-I$ space. Moreover, we are able to confirm kinematic membership of the cluster for 21 stars from an on-going radial velocity survey of the cluster (Meibom et al., in prep.), 5 of which show radial velocity variations (multiple systems). Four (4) of the photometric variables are kinematic non-members. Stars which are either photometric or kinematic non-members were excluded from the gyrochronology analysis.

We note the existence of another photometric period dataset for solar-type stars in the M 34 cluster (Irwin et al. 2006). We present an analysis comparing periods for stars in common between our Lowell campaign and their study. We detail several concerns that we have with the integrity of their dataset, 
notably their short observing window ( $<10$ nights) and their half-night observing cadence. After having verified that several Irwin et al. periods are most likely measurement aliases, and that our Lowell campaign periods agree those derived from an independent, separate, long time base-line differential photometry survey, we preferentially employ our period results in pursuing a gyrochronology age analysis for this cluster.

Rotation periods for bona fide cluster members, in concert with photometric $B-V$ colours, are used to create colour-period distributions, from which we outline a specific methodology for performing a gyrochronology analysis. In order to calculate the actual gyrochronology age of the cluster, we assign each M34 member its CgI status, as judged from its position in the colourperiod diagram. I-sequence stars in M 34 are classified specifically as those objects in the colour-period diagram lying above the I-sequence locus of the younger M35 cluster, yielding a mean gyro age for all I-sequence stars in M 34 of $193 \pm 9$ Myr.

There are two existing sets of age determination for the M 34 cluster, using the traditional isochrone fitting method and the white dwarf cooling time-scale. Together with gyrochronology, all three methods suffer from various forms of dependency on stellar models under-pinning their theoretical frameworks. Furthermore, both the isochrone fitting and white dwarf methods suffer from a dependence on (or an assumption of) cluster distance, while gyrochronology is free from distance as an input parameter.

The isochronal age of M34 is not that well defined (see Sect. 2 and Table 1), with a broad range in determined values. Unfortunately, the lack of observed giants in the cluster, and its broad turn-off locus in the $V / B-V$ colour magnitude diagram precludes a precise assessment of its isochronal age. Based on existing studies, the mean isochronal age of the cluster is $250 \pm 67$ Myr.

The white dwarf cooling time-scale age for M34 is also problematic because its result is based upon only five (5) objects whose membership of the cluster is not yet confirmed. Furthermore, the terminus of the white dwarf sequence has not yet been observed. In simple terms, the faintest, coolest, and therefore oldest white dwarfs have yet to be characterized, and the cluster's cooling time-scale age of $64.0 \pm 12.9$ Myr (Rubin et al. 2008) must be considered as a lower limit.

We conclude by noting that a study of M34 by Meibom et al., including both a multi-month differential photometry campaign and also a multi-year radial velocity membership and binarity campaign, similar to their prior work in $\mathrm{M} 35$, is in preparation. Their survey will yield several hundred new photometric periods of M 34 solar-type stars, the I- and C-sequences of which should be far more clearly defined than they are from our Lowell campaign results. Their dataset should allow them to better define the gyrochronology age of M 34, and we await their results eagerly.

Acknowledgements. This research has been supported by NSF grant AST0349075 (Vanderbilt University), which is gratefully acknowledged. This research has made extensive use of the WEBDA database, operated by the Institute for Astronomy at the University of Vienna, as well as the SIMBAD database, operated at CDS, Strasbourg, France. Insightful discussions with Jonathon Irwin concerning his original M 34 rotation period dataset are appreciated.

\section{References}

Baraffe, I., Chabrier, G., \& Gallardo, J. 2009, ApJ, 702, L27

Barnes, S. A. 2003, ApJ, 586, 464 [B03]

Barnes, S. A. 2007, ApJ, 669, 1167 [B07]
Barnes, S. A. 2009, The Ages of Stars, proceedings of the International Astronomical Union Symposium, ed. E. E. Mamajek, D. R. Soderblom, \& R. F. G. Wyse (Cambridge University Press), 258, 345

Barrado y Navascués, D., Stauffer, J. R., \& Patten, B. M. 1999, ApJ, 522, L53

Barry, D. C., Cromwell, R. H., Hege, K., \& Schoolman, S. A. 1981, ApJ, 247, 210

Basri, G., Marcy, G., \& Graham, J. 1996, ApJ, 458, 600

Beaudet, G., Petrosian, V., \& Salpeter, E. E. 1967, ApJ, 150, 979

Bedin, L. R., Salaris, M., Piotto, G., et al. 2005, ApJ, 624, 45

Bopp, B. W., \& Fekel, F. C. 1977, AJ, 82, 490

Caldwell, J. A. R., Cousins, A. W. J., Ahlers, C., van Wamelen, P., \& Maritz,

E. J. 1993, South African Astronomical Observatory Circulars, 15, P1

Canterna, R., Perry, C. L., \& Crawford, D. L. 1979, PASP, 91, 263

Cargile, P A., James, D. J., \& Platais, I. 2009, AJ, 137, 3230

Cayrel, R., Hill, V., Beers, T. C., et al. 2001, Nature, 409, 691

Cester, B., Giuricin, G., Mardirossian, F., \& Pucillo, M. 1977, A\&AS, 30, 227

Cox, J. P., \& Giuli, R. T. 1965, Principles of Stellar Structure (New York: Gordon \& Breach)

CRC Handbook of Chemistry and Physics, 86th edition, 2005 (Boca Raton: Taylor \& Francis)

D’Antona, F., \& Mazzitelli, I. 1989, ApJ, 347, 934

D’Antona, F., \& Mazzitelli, I. 1997, Mem. Soc. Astron. It., 68, 807

Demarque, P. R., \& Larson, R. B. 1964, ApJ, 140, 1544

Demarque, P. R., \& Gisler, G. R. 1975, A\&AS, 201, 237

Donahue, R. A., Saar, S. H. \& Baliunas, S. L. 1996, ApJ, 466, 384

Donati, J. F., Semel, M., Carter, B. D., Rees, D. E., \& Collier Cameron, A. 1997, MNRAS, 291, 658

Endal, A. S., \& Sofia, S. 1981, ApJ, 243, 625

Fekel, F. C., Moffet, T. J., \& Henry, G. W. 1986, ApJS, 60, 551

Fontaine, G., Brassard, P., \& Bergeron, P. 2001, PASP, 113, 409

Giampapa, M. S., Hall, J. C., Radick, R. R., \& Baliunas, S. L. 2006, ApJ, 651, 444

Gray, D. F. 1992, The Observation and Analysis of Stellar Photospheres, 2nd edn. (Cambridge: University Press)

Hall, D. S. 1976, Periodic Variable Stars, ed. W. Fitch (Dordrecht: Reidal), 287 Hambly, N. C., MacGillivray, H. T., Read, M. A., et al. 2001a, MNRAS, 326, 1279

Hambly, N. C., Davenhall, A. C., Irwin, M. J., \& MacGillivray, H. T. 2001b, MNRAS, 326, 1315

Hambly, N. C., Irwin, M. J., \& MacGillivray, H. T. 2001c, MNRAS, 326, 1295

Hartoog, M. R. 1977, ApJ, 212, 723

Henry, T. J., Soderblom, D. R., Donahue, R. A., \& Baliunas, S. L. 1996, AJ, 111, 439

Ianna, P. A. 1970, PASP, 82, 825

Ianna, P. A., \& Schlemmer, D. M. 1993, AJ, 105, 209

Iben, Jr, I., \& Laughlin, G. 1989, ApJ, 341, 312

Irwin, J., Aigrain, S., Hodgkin, S., et al. 2006, MNRAS, 370, 954

James, D. J., \& Jeffries, R. D. 1997, MNRAS, 291, 252

James, D. J., Melo, C., Santos, N., \& Bouvier, J. 2006, A\&A, 446, 971

Jeffries, R. D. 2009, The Ages of Stars, proceedings of the International

Astronomical Union Symposium, ed. E. E. Mamajek, D. R. Soderblom, \&

R. F. G. Wyse (Cambridge University Press), 258, 95

Jianke, L., \& Collier Cameron, A. 1993, MNRAS, 261, 766

Johnson, H. L. 1954, ApJ, 119, 185

Jones, B. F., \& Prosser, C. F. 1996, AJ, 111, 1193

Jones, B. F., Fischer, D., Shetrone, M., \& Soderblom, D. R. 1997, AJ, 114, 352

Kalirai, J. S., Fahlman, G. G., Richer, H., \& Ventura, P. 2003, AJ, 126, 1402

Kalirai, J. S., Richer, H. B., Reitzel, D., et al. 2005, ApJ, 618, 123

Kawaler, S. D. 1988, ApJ, 333, 236

Kawaler, S. D. 1989, ApJ, 343, 65

Koester, D., \& Schönberner, D. 1986, A\&A, 154, 125

Landolt, A. U. 1992, AJ, 104, 340

Landstreet, J. D., Silaj, J., Andretta, V., et al. 2008, A\&A, 481, 465

Maggio, A., Sciortino, S., Vaiana, G. S., et al. 1987, ApJ, 315, 687

Mamajek, E., \& Hillenbrand, L. 2008, ApJ, 687, 1264

Meibom, S. 2009, The Ages of Stars, proceedings of the International Astronomical Union Symposium, ed. E. E. Mamajek, D. R. Soderblom, \& R. F. G. Wyse (Cambridge University Press), 258, 357

Meibom, S., Mathieu, R., \& Stassun, K. 2006, ApJ, 653, 621

Meibom, S., Mathieu, R., \& Stassun, K. 2009, ApJ, 695, 679

Mengel, J. G., Sweigart, A. V., Demarque, P. R., \& Gross, P. G. 1979, ApJS, 40, 733

Mermilliod, J.-C., Grenon, M., Platais, I., James, D. J., \& Cargile, P. A. 2008, A\&A, 485, 95

Mestel, L. 1952, MNRAS, 112, 583

Meynet, G., Mermilliod, J.-C., \& Maeder, A. 1993, A\&AS, 98, 477

Naylor, T. 2009, MNRAS, 399, 432 
Noyes, R. W., Hartmann, L. W., Baliunas, S. L., Duncan, D. K., \& Vaughan, A. H. 1984, ApJ, 279, 763

Palla, F., \& Randich, S. 2005, The Initial Mass Function 50 Years Later, INAF Osservatorio Astrofisico di Arcetri, Firenze, Italy, ed. E. Corbelli, \& F. Palle (Dordrecht: Springer), Astrophys. Space Sci. Libr., 327, 73

Perryman, M. A. C., Brown, A. G. A., Lebreton, Y., et al. 1998, A\&A, 331, 81

Prosser, C. F., Stauffer, J. R., Caillault, J.-P., et al. 1995a, AJ, 110, 1229

Prosser, C. F., Shetrone, M. D., Dasgupta, A., et al. 1995b, PASP, 107, 211

Raboud, D., \& Mermilliod, J. C. 1998, A\&A, 329, 101

Radick, R. R., Thompson, D. T., Lockwood, G. W., Duncan, D. K., \& Baggett, W. E. 1987, ApJ, 321, 459

Rebolo, R., Martín, E. L., Basri, G., \& Zapatero-Osorio, M. R. 1996, ApJ, 469, L53

Richer, H. B., Fahlman, G. G., Rosvick, J., \& Ibata, R. 1998, ApJ, 504, 91

Roberts, D. H., Lehar, J., \& Dreher, J. W. 1987, AJ, 93, 968

Rubin, K. H. R., Williams, K. A., Bolte, M., \& Koester, D. 2008, AJ, 135, 2163

Sandage, A. 1958, Specola Vaticana, Proceedings of a Conference at Vatican Observatory, Castel Gandolfo, May 20-28, 1957, ed. D. J. K. O'Connell (Amsterdam, North-Holland, and New York: Interscience), Ricerche Astron., 5,41

Skumanich, A. 1972, ApJ, 171, 565

Soderblom, D. R., Duncan, D. K., \& Johnson, D. R. H. 1991, ApJ, 375, 722

Soderblom, D. R., Stauffer, J. R., MacGregor, K. B., \& Jones, B. F. 1993a, ApJ, 409,624
Soderblom, D. R., Jones, B. F., Balachandran, S., et al. 1993b, AJ, 106, 1059 Soderblom, D. R., Stauffer, J. R., Hudon, J. D., \& Jones, B. F. 1993c, Ap\&SS, 85,315

Soderblom, D. R., Jones, B. F., \& Fischer, D. 2001, ApJ, 563, 334

Stauffer, J. R. 1984, ApJ, 280, 189

Stauffer, J. R., Hartmann, L. W., Soderblom, D. R., \& Burnham, N. 1984, ApJ, 280,202

Stauffer, J. R., Schultz, G., \& Kirkpatrick, J. D. 1998, ApJ, 499, L199

Stetson, P. B. 1992, in Stellar Photometry - Current Techniques and Future Developments, ed. C. J. Butler, \& I. Elliot, IAU Colloq., 136, 291

Stetson, P. B., Davis, L. E., \& Crabtree, D. R. 1991, in CCDs in Astronomy, ed. G. Jacoby, ASP Conf. Ser., 8, 282

Wichmann, R., Torres, G., Melo, C. H. F., et al. 2000, A\&A, 359, 181

Wilson, O. C. 1963 , ApJ, 138, 832

Wilson, O. C. 1964, PASP, 76, 28

Wilson, O. C. 1966 , Science, 151, 1487

Wilson, O. C., \& Skumanich, A. 1964, ApJ, 140, 1401

Wood, M. A. 1992, ApJ, 386, 539

Zahn, J.-P. 1989, A\&A, 220, 112

Zahn, J.-P. 1994, A\&A, 288, 829

Zombeck, M. V. 1990, Handbook of Space Astronomy and Astrophysics, 2nd edn. (Cambridge: University Press) 


\section{J. James et al.: The gyrochronology age of M34}

\section{Appendix A: Cluster membership of M 34 Using extant kinematic measurements}

There exist two extant kinematic studies of the M34 cluster which can assist us further in establishing cluster membership for our gyrochronology sample. The first, by Jones \& Prosser (1996), relies upon 2-d space motions in the form of proper motions, although their high-fidelity membership probabilities are magnitude limited to about $V \simeq 14.5$. While JP96 proper motion results do extend to fainter magnitudes, their reliability reduces. That is to say, a fainter M 34 star with a $00 \%$ proper motion probability may indeed still be a bona fide member of the cluster, insomuch as Poisson-limited proper motion errors could lead to erroneously lower proper motion probabilities.

The second, by Jones et al. (1997), employing 1-d heliocentric radial velocities, can be used to establish cluster membership, or in the very least, establish cluster non-membership (or binarity). Unfortunately neither membership probabilities nor velocity errors were reported by Jones et al., however we can exploit their measurements in our membership assessment (see below). Interestingly, in the same manuscript, Jones et al. report Li I $6708 \AA$ equivalent widths [EWs] for their target stars. Even though the detection of lithium in solar type stars is not a requisite for cluster membership, its absence is almost ubiquitous among the generally older, Galactic field stars. The very presence of a substantial lithium line in solar-type stellar spectra is in itself indicative of youth $(\ll 1000 \mathrm{Myr}$; e.g., Soderblom et al. 1993b; James \& Jeffries 1997; James et al. 2006).

For each of the M34 stars in our sample, we have collated these additional membership criteria published by JP96 and Jones et al. (1997) in Table A.1. Its Lowell identifier is listed in Col. 1 in concert with its JP96 identifier in Col. 2. Each star's photometric and radial velocity membership flag, reproduced from Tables 2 and 3, are cited in Cols. 3 and 4 respectively, whereas a flag confirming the detection of lithium $6708 \AA$ in its spectrum (from Jones et al. 1997) is listed in Col. 5. Corresponding Jones et al. radial velocities are detailed in Col. 6, as well as their associated membership probabilities in Col. 7 (see below and Fig. A.1). $V$-band magnitudes of each star are listed in Col. 8 and finally, cluster membership probabilities based on the proper motions reported in JP96 are reproduced in Col. 9.

In the first instance, we note that all stars in common between our gyrochronology sample and the Jones et al. sample have a significant lithium detection, indicative of stellar youth. These stars are therefore unlikely to be field star interlopers, which when combined with the photometric membership criterion, can safely be considered as bona fide cluster members. In the second, the radial velocity data from Jones et al. study do seem to cluster around $-10 \mathrm{~km} \mathrm{~s}^{-1}$, however, specific membership assignments are difficult to assess due to the associated scatter in the individual measurements. In order to understand whether the scatter in these radial velocities is due to a true dispersion in the data or is an admixture of single and binary member measurements, together with cluster non-members, we have constructed a histogram for all radial velocities published in the Jones et al. survey. This histogram, plotted in Fig. A.1, clearly shows a peak in the distribution representing the cluster's systemic heliocentric radial velocity. In order to investigate the dispersion about the central peak, we fit an unweighted Gaussian function to the velocity histogram, noting that the fit does not include background contamination due to binarity or cluster non-members, and assumes that the sample is complete. The Gaussian function is centred on $-12.49 \mathrm{~km} \mathrm{~s}^{-1}$ with a $1 \sigma$ width of $2.32 \mathrm{~km} \mathrm{~s}^{-1}$, which we can exploit to determine a membership probability (reported in Col. 7 of Table A.1) for each individual star based on its radial velocity and these Gaussian fit parameters.

\section{A.1. Results}

Analysis of the additional membership data reported in Table A.1 yields few surprises. Of all the Lowell periodic variables identified as photometric and/or RV members in Table 2, only two of them, F3_0172 and F3_0215, have a zero probability of being proper motion members according to the JP96 survey. Interestingly however, their $V$-magnitudes lie right at the point at which the JP96 proper motions begin to become less reliable due to Poisson-error limits on their photographic plate measurements, which brings their validity into question. Furthermore, we have noted in Sect. 5.2 that the gyrochronology sample may indeed suffer from contamination due to one or two cluster nonmembers masquerading as bona fide members, but we argue that their contribution to the period variance, and hence gyro errors, is small. In fact, F3_0172 and F3_0215 contribute a combined period variance of 0.79 days $^{2}$, which is only a $1.8 \%$ effect (see Table 5). In spite of their low contribution to the period variance, we remain in the uncomfortable position of choosing whether to include these photometric and kinematic members of the cluster in our gyrochronology sample, or to exclude them on the basis of their proper motion membership probabilities. In order to further assess their cluster membership status, we have recently obtained high resolution optical spectra of these two stars using the Canada France Hawai' $i$ Telescope [CFHT], the analysis of which we discuss below (see Sect. A.2).

Finally, all bar one of the Jones et al. (1997) stars are radial velocity members of the cluster, which correlates well with our own radial velocity data. The one Jones et al. star, F4_0136, that is formally a cluster non-member based on a Gaussian probability fit to their radial velocity data plotted in Fig. A.1, actually shows up as a single cluster star member in the long term synoptic velocity survey of Meibom et al. (in prep. - see also Sect. 2.1). Curiously, over the 9-epochs of observation covering 2.5-years, that Meibom et al. have for this star, its varies about their average radial velocity of $-8.0 \mathrm{~km} \mathrm{~s}^{-1}$ by only $0.68 \mathrm{~km} \mathrm{~s}^{-1}$. Interestingly, a Gaussian fit to all radial velocities for the 70 single and binary M 34 stars for which Meibom et al. have obtained results yields a cluster systemic velocity of $-7.59 \pm 1.02 \mathrm{~km} \mathrm{~s}^{-1}$. Assuming the Jones et al. velocity is not in error, this star may be a binary member of the cluster, albeit with either a long-period orbit or a considerably eccentric one.

\section{A.2. Cluster membership status for stars F3_0172 and F3_0215:}

Two stars exhibiting variability in our differential photometric survey of the M34 cluster, for which we derive periods, namely F3_0172 and F3_0215, present us with somewhat of a conundrum. While these stars have photometric and radial velocity properties consistent with cluster membership, with relatively short photometric periods indicative of youth (compared to the Galactic field), and result in gyrochronology ages appropriate for a 200 Myr group of stars, they possess proper motion vectors incongruent with the remainder of the cluster. In an attempt to establish or refute genuine cluster membership for these two objects, we have recently observed them at high resolution using the fibre-fed, bench-mounted ESPaDOns échelle spectrograph, located in a Coudé-like instrument chamber in the 
A\&A 515, A100 (2010)

Table A.1. Cluster membership assessments for Jones \& Prosser (1996) stars in our period sample.

\begin{tabular}{|c|c|c|c|c|c|c|c|c|}
\hline $\begin{array}{l}\text { Internal }^{a} \\
\text { Identifier }^{2}\end{array}$ & JP96 ${ }^{b}$ & $\begin{array}{l}\mathrm{Phot}^{a} \\
\text { Mem }\end{array}$ & $\begin{array}{l}\mathrm{RV}^{a} \\
\mathrm{Mem}\end{array}$ & $\begin{array}{l}\mathrm{Li} \mathrm{Mem}^{c} \\
\text { J97 }\end{array}$ & $\begin{array}{l}\mathrm{RV} \mathrm{J} 97^{c} \\
{\left[\mathrm{~km} \mathrm{~s}^{-1}\right]}\end{array}$ & $\begin{array}{c}\mathrm{RV} \mathrm{Mem} \\
\mathrm{J} 97\end{array}$ & $\begin{array}{c}\mathrm{V}^{a} \\
{[\mathrm{mag}]}\end{array}$ & $\begin{array}{c}\text { PM Prob }^{e} \\
\text { JP96 }\end{array}$ \\
\hline F3 0172 & JP 49 & $\mathrm{Y}$ & Ys & $\ldots$ & t... & $\ldots$ & 14.584 & $00 \%$ \\
\hline F3_0176 & JP 167 & Y & Ys & $\ldots$ & $\ldots$ & $\ldots$ & 14.659 & $44 \%$ \\
\hline F3_0215 & JP 41 & $\mathrm{Y}$ & Ys & $\ldots$ & $\ldots$ & $\ldots$ & 14.918 & $00 \%$ \\
\hline F3_0258 & JP 289 & Y & Ys & $\dddot{Y}$ & $\begin{array}{l}\cdots \\
-10.8\end{array}$ & Y $(\dddot{47 \%})$ & 15.236 & $76 \%$ \\
\hline F3_0306 & JP 265 & Y & $\mathrm{Yb}$ & Y & -17 & $Y(5 \%)$ & 15.503 & $53 \%$ \\
\hline F3_0320 & JP 172 & Y & $\ldots$ & Y & -9.8 & $Y(25 \%)$ & 15.664 & $03 \%$ \\
\hline F4_0136 & JP 213 & $\mathrm{Y}$ & Ys & $\mathrm{Y}$ & -5.7 & $\mathrm{~N}(0 \%)$ & 13.151 & $94 \%$ \\
\hline F4_0169 & JP 50 & Y & Ys & $\ldots$ & $\ldots$ & $\ldots$ & 13.510 & $04 \%$ \\
\hline F4_0194 & JP 133 & $\mathrm{Y}$ & Ys & Y & -11.8 & Y $(77 \%)$ & 13.662 & $96 \%$ \\
\hline $\mathrm{F} 40234$ & JP 148 & Y & Ys & $\ldots$ & $\ldots$ & ... & 13.959 & $05 \%$ \\
\hline F4_0303 & JP 227 & Y & Ys & Y & -15.4 & $\mathrm{Y}(21 \%)$ & 14.366 & $92 \%$ \\
\hline F4_0317 & JP 224 & Y & Ys & Y & -9.4 & $\mathrm{Y}(18 \%)$ & 14.410 & $91 \%$ \\
\hline F4_0327 & JP 199 & Y & Ys & Y & -9.0 & $Y(13 \%)$ & 14.483 & $85 \%$ \\
\hline F4_0335 & JP 158 & Y & $\mathrm{Yb}$ & Y & -9.9 & $\mathrm{Y}(26 \%)$ & 14.546 & $86 \%$ \\
\hline F4_0450 & JP 113 & Y & Ys & Y & -13.5 & $Y(67 \%)$ & 14.960 & $84 \%$ \\
\hline F4_0667 & JP 52 & $\mathrm{Y}$ & $\mathrm{Yb}$ & $\ldots$ & $\ldots$ & $\ldots$ & 15.623 & $26 \%$ \\
\hline F4 0695 & JP 197 & Y & Ys & $\ldots$ & $\ldots$ & $\ldots$ & 15.643 & $05 \%$ \\
\hline F4_0730 & JP 18 & $\mathrm{Y}$ & $\ldots$ & $\cdots$ & $\begin{array}{l}\cdots \\
\ldots\end{array}$ & $\ldots$ & 15.741 & $36 \%$ \\
\hline F4_0803 & JP 229 & Y & Ys & $\mathrm{Y}$ & -9.9 & $Y(26 \%)$ & 16.025 & $46 \%$ \\
\hline F3_0121 & JP 212 & $\mathrm{~N}$ & & $\ldots$ & $\ldots$ & $\ldots$ & 13.741 & $00 \%$ \\
\hline F3_0163 & JP 258 & Y & $\dddot{N}$ & $\cdots$ & $\cdots$ & $\cdots$ & 14.440 & $00 \%$ \\
\hline F3_0165 & JP 244 & Y & $\mathrm{N}$ & $\ldots$ & $\ldots$ & $\ldots$ & 14.419 & $00 \%$ \\
\hline F3_0388 & JP 174 & $\mathrm{~N}$ & $\ldots$ & $\ldots$ & $\ldots$ & $\ldots$ & 15.952 & $00 \%$ \\
\hline F3_0413 & JP 218 & $\mathrm{Y}$ & $\mathrm{N}$ & $\ldots$ & $\ldots$ & $\ldots$ & 16.242 & $17 \%$ \\
\hline F4_0167 & JP 223 & $\mathrm{~N}$ & $\ldots$ & $\ldots$ & $\ldots$ & $\ldots$ & 13.502 & $00 \%$ \\
\hline F4 0404 & JP 131 & Y & $\mathrm{N}$ & $\ldots$ & $\ldots$ & $\ldots$ & 14.730 & $00 \%$ \\
\hline F4_0515 & JP 129 & $\mathrm{~N}$ & $\ldots$ & $\ldots$ & $\ldots$ & $\ldots$ & 15.111 & $00 \%$ \\
\hline
\end{tabular}

Notes. ${ }^{(a)}$ Data taken from Tables 2 and 3; ${ }^{(b)} J P$ identifier from Jones \& Prosser (1996); ${ }^{(c)}$ cluster membership based on radial velocities and lithium $6708 \AA$ A equivalent widths, taken from Jones et al. (1997) [J97]; ${ }^{(d)}$ radial velocity membership assessments are based on a Gaussian fit to the entire Jones et al. (1997) radial velocity sample (see also Fig. A.1); ${ }^{(e)}$ proper motion membership probabilities are taken from JP96.

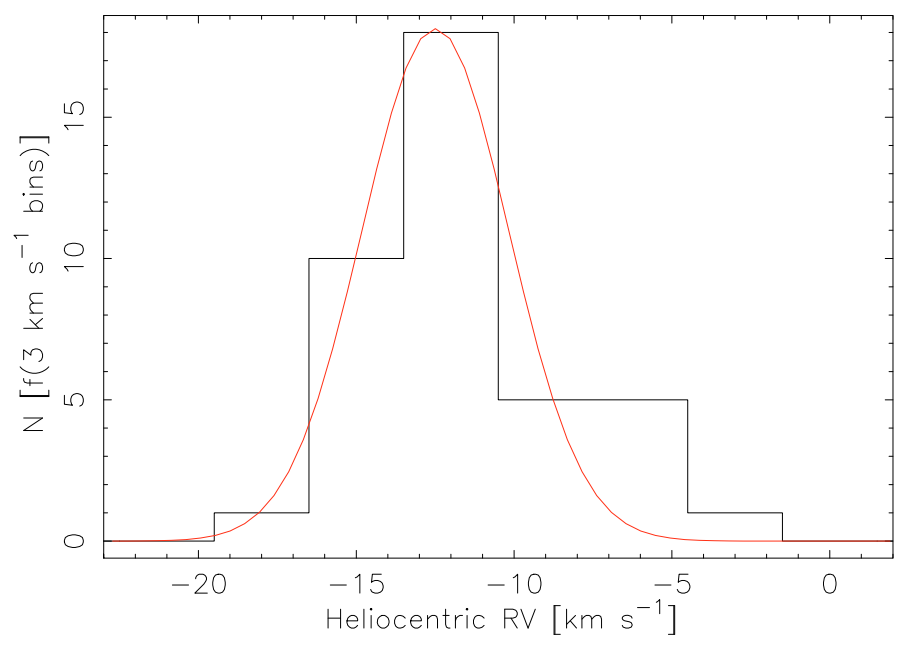

Fig. A.1. Heliocentric radial velocities, in histogram form using $3 \mathrm{~km} \mathrm{~s}^{-1}$ bins, are plotted for M 34 stars using velocity data detailed in Jones et al. (1997). The red solid line depicts an unweighted Gaussian fit to the data, with a Gaussian centre of $-12.49 \mathrm{~km} \mathrm{~s}^{-1}$ and a sigma of $2.32 \mathrm{~km} \mathrm{~s}^{-1}$.

CFHT Observatory. The primary goal of these observations is to detect lithium at $6708 \AA$, and measure its equivalent width in these stars, thereby confirming their relative youth and increasing their probability of being bona fide cluster members.

During the evening of 27 January 2010, high resolution $(R \simeq 60000)$ spectra were acquired for stars F3_0172 and
F3_0215 using the fibre-fed ESPaDOns spectrograph, in service with the 3.6-m Canada France Hawai“i Telescope located on top of Mauna Kea, Hawai 'i, USA. The ESPaDOns spectrograph consists of a 79 lines $\mathrm{mm}^{-1}$ échelle grating imaged onto a $2048 \times 4608$ EEV CCD detector, having $13.5 \mu \mathrm{m}$ square pixels, with photon input delivered by separate $100 \mu \mathrm{m}$ (1.6 arcsec) diameter sky and target fibres. This set-up yields a FWHM of cross correlated ThAr arc lines of $0.271 \AA$ at $6700 \AA$, and a complete wavelength range of $3699 \rightarrow 10481 \AA$. Using this setup, F3_0172 and F3_0215 were observed for exposures totalling 2000 and 2140 s respectively, resulting in spectra with $S / N \simeq 20$ at $6700 \AA$.

For each of the targets, we exploit our CFHT ESPaDOns spectra in order to measure their heliocentric radial velocity and their Li I $6708 \AA \mathrm{A} W$. The removal of CCD instrumental effects, as well as the extraction of wavelength-calibrated spectra, have been achieved by two independent data reduction methodologies. In the first, we performed the bias-subtraction, flat-fielding, spectral order tracing, optimal extraction and wavelength calibration using standard IRAF procedures. In the second, we used the direct output from Libre-ESpRIT (Donati et al. 1997), the dedicated pipeline software for the ESPaDOns spectrograph. A cross-match of radial velocities and $E W$ s for each target from the two reduction methods reassuringly yields consistent results to within $\pm 0.1 \mathrm{~km} \mathrm{~s}^{-1}$ and $\pm 3 \mathrm{~m} \AA$ respectively.

Heliocentric radial velocities were derived, relative to the well-exposed standard star HD 32963, in the spectral region of the $\mathrm{Mg}$ triplet lines $(5104 \rightarrow 5176 \AA)$. Cross correlation of HD 32963 with other radial velocity standard stars observed 
D. J. James et al.: The gyrochronology age of M 34

Table A.2. CFHT spectroscopic data products for M 34 candidate members F3_0172 and F3_0215.

\begin{tabular}{|c|c|c|c|c|c|c|c|c|c|}
\hline $\begin{array}{l}\text { Internal }^{a} \\
\text { Identifier }^{2}\end{array}$ & $\mathrm{JP}^{2} 6^{b}$ & $(B-V)_{o}{ }^{a}$ & $\begin{array}{c}\text { Temp }^{c} \\
{[\mathrm{~K}]} \\
\end{array}$ & $\begin{array}{c}\mathrm{HJD}^{d} \\
{[(+2450000)]}\end{array}$ & $\begin{array}{c}\mathrm{RV} \\
{\left[\mathrm{km} \mathrm{s}^{-1}\right]}\end{array}$ & $\begin{array}{c}\mathrm{Li} \mathrm{I}+\mathrm{Fe} \mathrm{I}^{e} \\
E W[\mathrm{~m} \AA]\end{array}$ & $\begin{array}{c}\mathrm{Fe} \mathrm{I}^{f} \\
E W[\mathrm{m \AA}]\end{array}$ & $\begin{array}{c}\mathrm{Li} \text { I } 6708 \AA^{g} \\
E W[\mathrm{~mA}]\end{array}$ & $N(\mathrm{Li})^{h}$ \\
\hline F3_0172 & JP 49 & 0.811 & 5139 & 5223.713 & -8.4 & 115 & 13 & 102 & 2.003 \\
\hline F3_0215 & JP 41 & 0.874 & 4946 & 5223.712 & -7.4 & 153 & 14 & 139 & 1.985 \\
\hline
\end{tabular}

Notes. ${ }^{(a)}$ Data taken from Tables 2 and $3 ;^{(b)} J P$ identifier from Jones \& Prosser (1996); ${ }^{(c)}$ temperature determined from $(B-V)_{o}$ relationship in Soberblom et al. (1993c - Eq. (3)); ${ }^{(d)}$ HJD - heliocentric julian date; ${ }^{(e)}$ equivalent width measurement, by Gaussian fitting, of the blended Li I and Fe I 6708 A lines; ${ }^{(f)}$ Fe I $6707.441 \AA$ A line contribution, determined by an empirical $(B-V)$ colour fit (see Soderblom et al. $1993 \mathrm{~b}$ ); ${ }^{(g)}$ Li I $6708 \AA$ minus the Fe I $6707.441 \AA$ A contribution; ${ }^{(h)}$ Lithium equivalent widths are transformed into abundance $[N(\mathrm{Li})]-$ on a scale where $\log N(\mathrm{H})=12$, using a curve of growth in Soderblom et al. (1993b).

during the CFHT programme shows that the zero-point in placing our velocities onto the standard system is $\simeq 0.2 \mathrm{~km} \mathrm{~s}^{-1}$. The relatively low $S / N$ of our target spectra results in radial velocity precision errors of $\simeq 0.5 \mathrm{~km} \mathrm{~s}^{-1}$.

In order to measure equivalent widths for the Li I $6708 \AA$ line in our targets, each spectrum between $6586 \rightarrow 6711 \AA$, was normalized using continuum fitting after spectra extraction. Each $E W$ was calculated using both the direct integration and the Gaussian fitting methods, whose values were within a few percent of each other. In the lithium region, Li I EWs include contributions from the small Fe I+CN line at $6707.44 \AA$, leading to measured $\mathrm{LiI} E W \mathrm{~s}$ which are representative of a

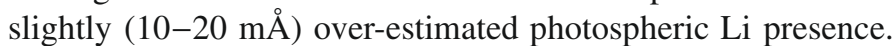
Soderblom et al. (1993b) report that this Fe line blend has an $E W=\left[20(B-V)_{0}-3\right] \mathrm{m} \AA$, determined through an empirical relationship for main sequence, solar-type stars. For each target star, we removed the Fe I line contribution before transforming Li I $E W$ s into abundances, $N(\mathrm{Li})-$ on a scale where $\log N(\mathrm{H})=12$, using the effective temperature-colour $(B-V)$ relation from Soderblom et al. (1993c), and the curves of growth presented in Soderblom et al. (1993b). Data products, radial velocity and lithium measurements, from the CFHT spectroscopic observations of F3_0172 and F3_0215 are presented in Table A.2.

CFHT radial velocities of F3_0172 and F3_0215 are consistent with cluster membership of $\bar{M} 34$ irrespective of whether we compare their individual values to the Jones et al. (1997) sample or to the Meibom et al. (in prep.) one. For the Jones et al. sample, their kinematic membership probabilities are non-zero although they are quite low at $08 \%$ and $03 \%$ (for F3_0172 and F3_0215 respectively). Their membership probabilities are far more convincing when compared to the Meibom et al. sample (with respective values of $79 \%$ and $>99 \%$ ).

The lithium content of both F3_0172 and F3_0215 is substantial indicating that these stars are relatively young compared to the general Galactic field, whose solar-type stellar content is typically old and has had sufficient time to have proton-burned considerable fractions of its natal lithium. In order for these stars to be judged as likely members of the M 34 cluster, not only must they contain lithium in their atmospheres, but it must quantitatively fit into the mass-dependent lithium abundance distribution for the cluster. In Fig. A.2, we plot the lithium abundances for F3_0172 and F3_0215 that we have determined from our CFHT spectra in concert with the extant mass-dependent lithium distribution for M 34 stars (data taken from Jones et al. 1997, who employed identical $(B-V)_{o}$-Temperature, Fe I line correction and lithium curves of growth as we have used). It is clear that both F3_0172 and F3_0215 have lithium abundances consistent with the mass-dependent lithium distribution of the M 34 cluster, and must be considered lithium abundance members.

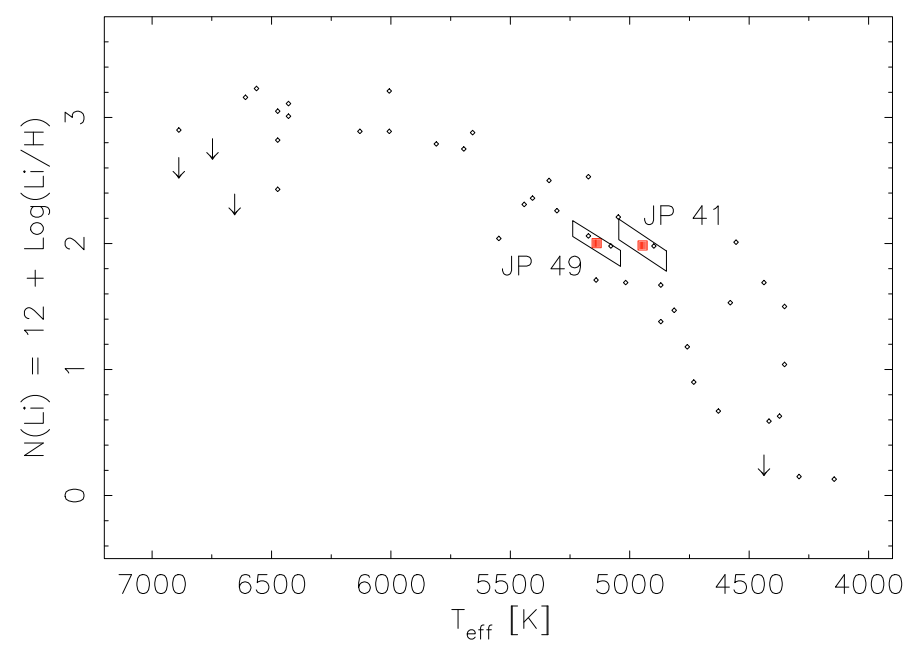

Fig. A.2. Logarithmic lithium abundances $N(\mathrm{Li})=12+\log (\mathrm{Li} / \mathrm{H})$ versus effective temperature are plotted for stars in the M 34 cluster (data taken from Jones et al. 1997). Red filled squares represent abundances for F3_0172 and F3_0215 (JP 49 and JP 41), determined using data reported in Table A.2, with error parallelograms based on temperature errors of $100 \mathrm{~K}$ and $15 \%$ equivalent width uncertainties.

JP96 proper motion vectors for F3_0172 and F3_0215 indicate cluster non-membership. However their $V$-magnitudes render JP96 2-d kinematic membership probabilities questionable, because at or about this magnitude, JP96 proper motion accuracy and precisions begin to have strong dependencies on the Poissonian errors of their centroiding measurements. In consideration that these two stars are both photometric and kinematic members of the cluster, have photometric periods consistent with the remainder of the cluster's distribution, and have measured lithium abundances which lie right along the trend of the massdependent lithium distribution for the cluster, they are probable M 34 cluster members and we retain them in our gyrochronology sample.

\section{Appendix B: A period vs. equatorial velocity analysis}

A comparison of photometric period and projected equatorial rotation rate for M34 stars is plotted in Fig. B.1. For the most part, photometric periods are determined from the Lowell campaign described in this manuscript, except for a few cases where Irwin et al. periods are employed where Lowell ones do not exist. Spectroscopic velocities for stars in common to the Lowell campaign and Irwin et al. survey are obtained from Jones et al. (1997). Three loci are also shown in the figure, 


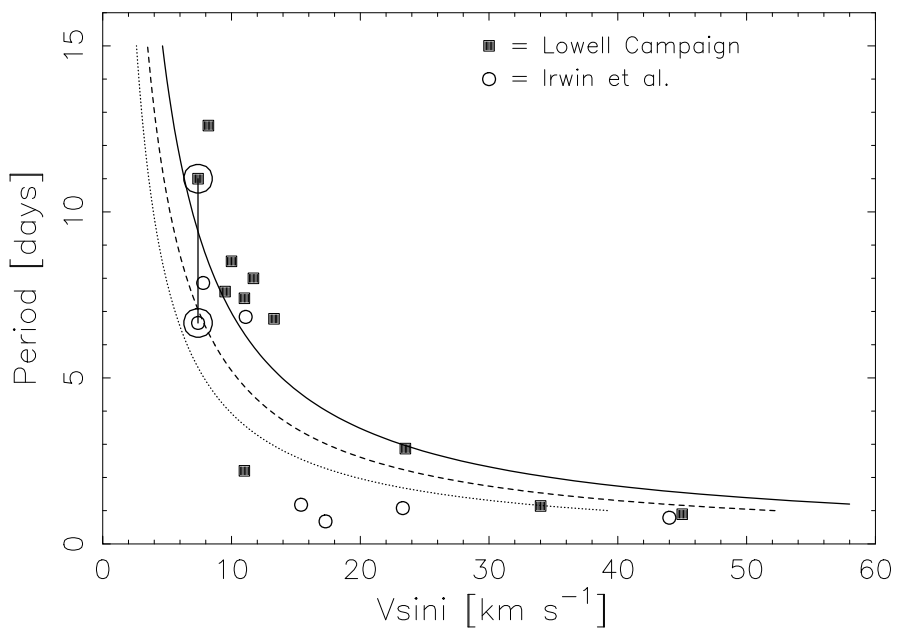

Fig. B.1. Projected equatorial velocities $(v \sin i)$ are plotted for those M 34 stars having corresponding photometric periods derived during the course of the Lowell campaign. $V \sin i$ data are taken from Jones et al. (1997). For those cases where there is no Lowell campaign period available, similar data from the Irwin et al. (2006) sample are presented. One exception is shown for the star F3_0258, linked by a straight solid line, whose Lowell campaign and Irwin et al. periods are seriously discrepant $(11.0 \pm 1.0$ and 6.655 days respectively). Three loci are depicted representing equal period-vs- $v \sin i$ relationships (assuming $\sin i=\pi / 4$ ) for G0, K0 and M0 dwarfs (solid, dashed and dotted lines respectively). In order of descending mass, stellar radii of $R / R_{\odot}=1.08,0.81$ and 0.61 were employed (Gray 1992).

representing equality between period and $v \sin i$ data for $\mathrm{G} 0, \mathrm{~K} 0$ and M0 dwarfs, where $\sin i=\pi / 4$ is assumed for each.

There are three notable characteristics to the plot. First, the clustering of data-points for stars with periods $\simeq 6.5 \rightarrow 9$ days and $v \sin i \simeq 10-14 \mathrm{~km} \mathrm{~s}^{-1}$ lie to the right of the G0 dwarf locus. Given that the M 34 stars having photometric periods are late-F to early M-dwarfs, these data-points are most likely indicative of those stars whose $\sin i$ values are $>\pi / 4$. Second, there are four stars with periods $<3$ days and $10<v \sin i<25 \mathrm{~km} \mathrm{~s}^{-1}$, whose period, $v \sin i$ data place them considerably below even the M0 dwarf locus in the diagram. Assuming that these stars are bona fide cluster members, whose period and $v \sin i$ values are correctly determined, these stars must be high inclination systems ( $\sin i<\pi / 4$; i.e., becoming more pole-on), whose true equatorial velocities are considerably higher.
Finally, there is one M34 star (F3_0258) whose period determinations, from our Lowell data and by the Irwin et al. study, are seriously discrepant (see also Fig. 2). The periodicity for this star was detected in three filter/exposure time observations during our Lowell campaign. It is interesting to note that the Irwin et al. period is almost half that of our Lowell one, and their lower value could be due to phase aliasing, power leakage in the power spectrum as a consequence of their shorter observing window or multiple spot groups on the surface on the star during its observation. In any case, either both period determinations are incorrect, or either value from the Lowell campaign or the Irwin et al. study is in error.

If we assume that one of the periods for this star is correct, we can make some predictive statements as to its equatorial velocity. With an intrinsic $B-V$ colour of 0.95 , its spectral type on the main sequence would be $\mathrm{K} 2$ or $\mathrm{K} 3$, placing it close to the central locus of the three plotted in Fig. B.1 (assuming the star is inclined $45^{\circ}$ to the line-of-sight). This scenario is consistent with its Irwin et al. period of 6.655 days. Conversely, if the Lowell period of 11.0 days is correct for this object, and it is a single member of the cluster lying on the main sequence, its inclination angle must be higher than $45^{\circ}(\sin i>\pi / 4)$, whose appearance is more face-on to the line-of-sight. Hopefully, more extensive photometric monitoring of this star will reveal its true nature.

\section{Appendix C: Lowell light-curves for photometrically variable stars in the field of M 34}


D. J. James et al.: The gyrochronology age of M 34

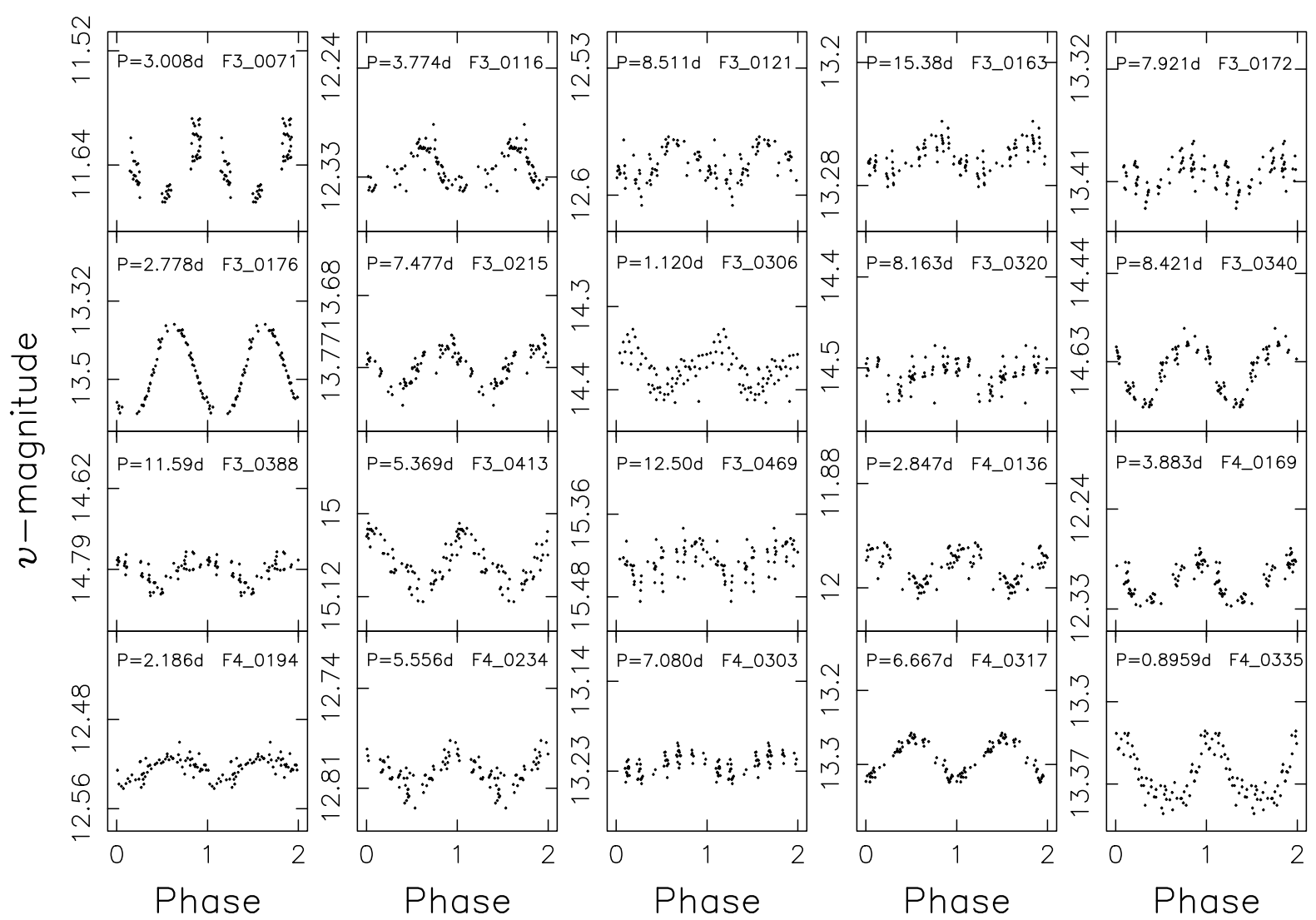

Fig. C.1. Period-phased photometric lightcurves for M 34 variables, derived from short-V observations.

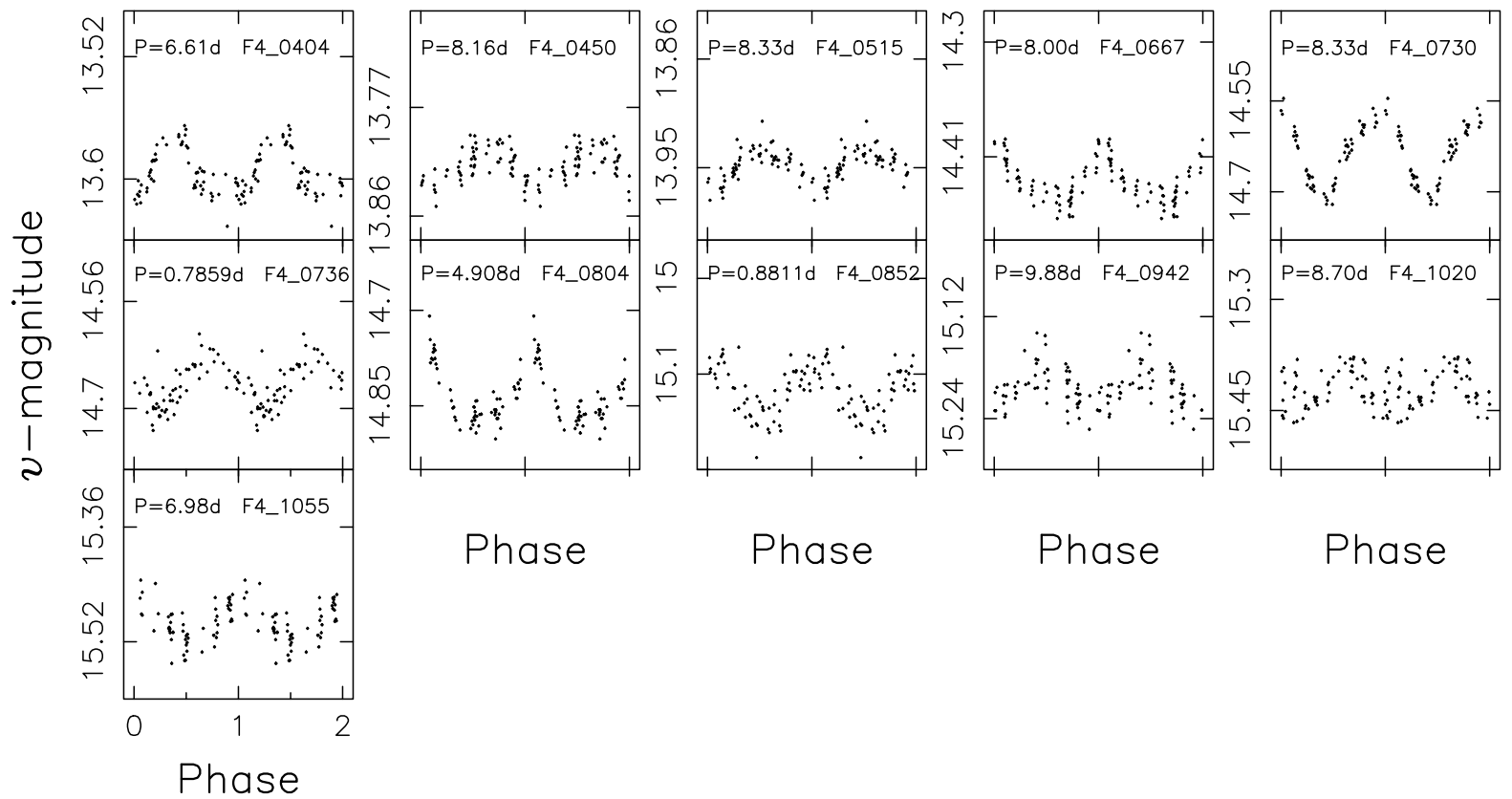

Fig. C.1. continued. 
A\&A 515, A100 (2010)

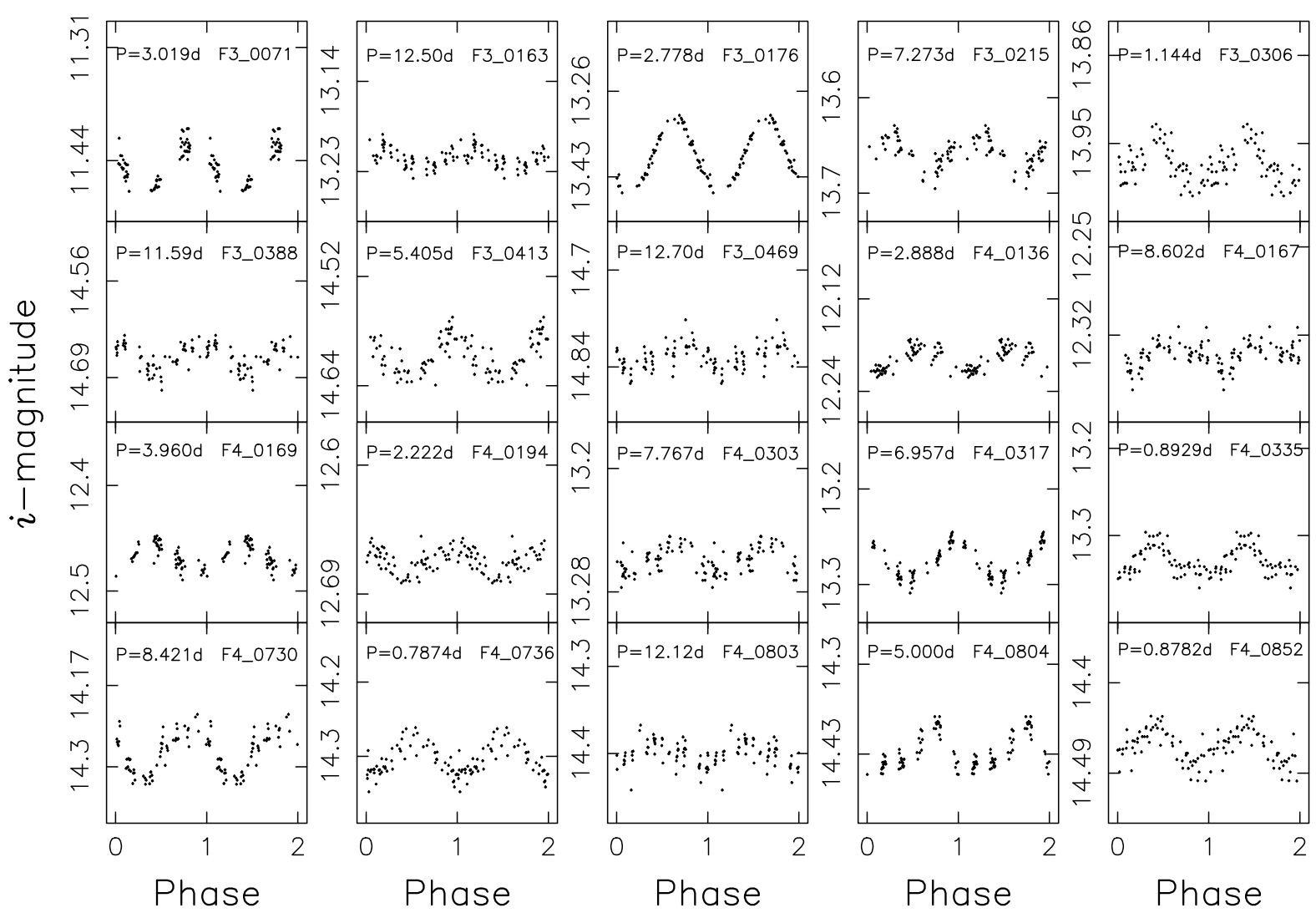

Fig. C.2. Period-phased photometric lightcurves for M 34 variables, derived from short-I observations.

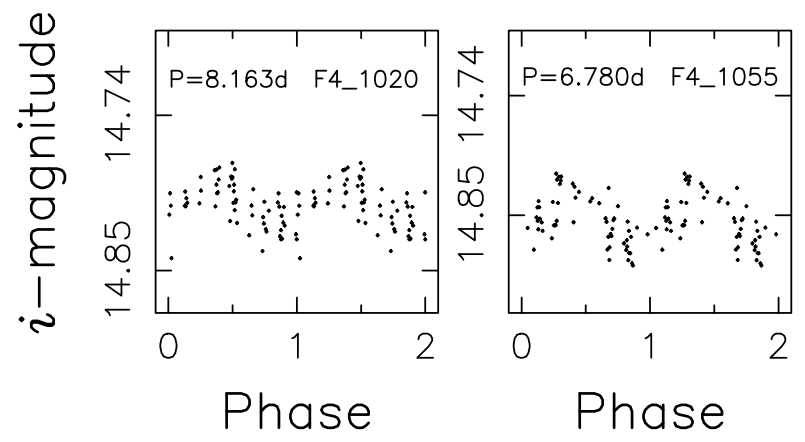

Fig. C.2. continued. 
D. J. James et al.: The gyrochronology age of M 34

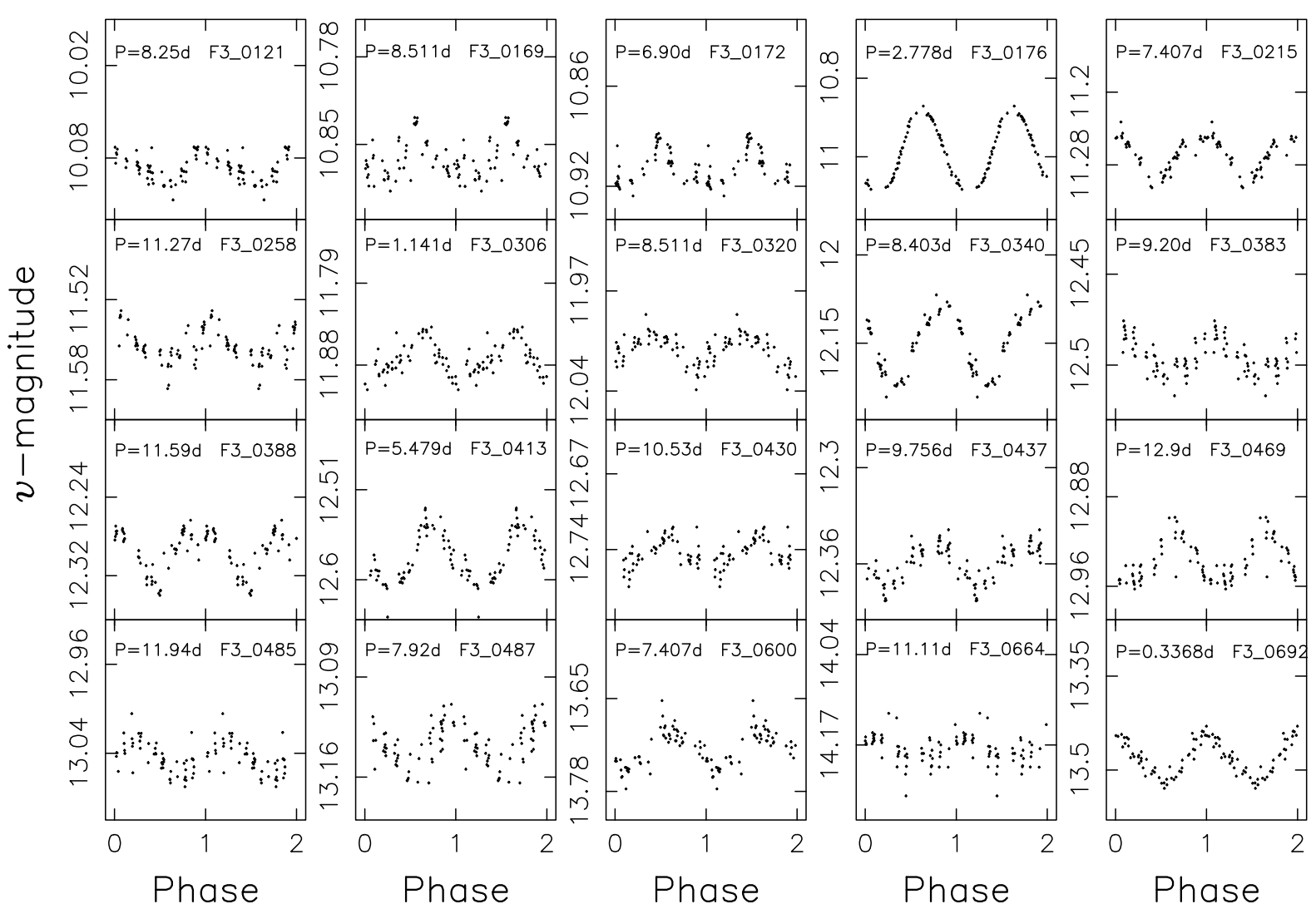

Fig. C.3. Period-phased photometric lightcurves for M 34 variables, derived from long-V observations.

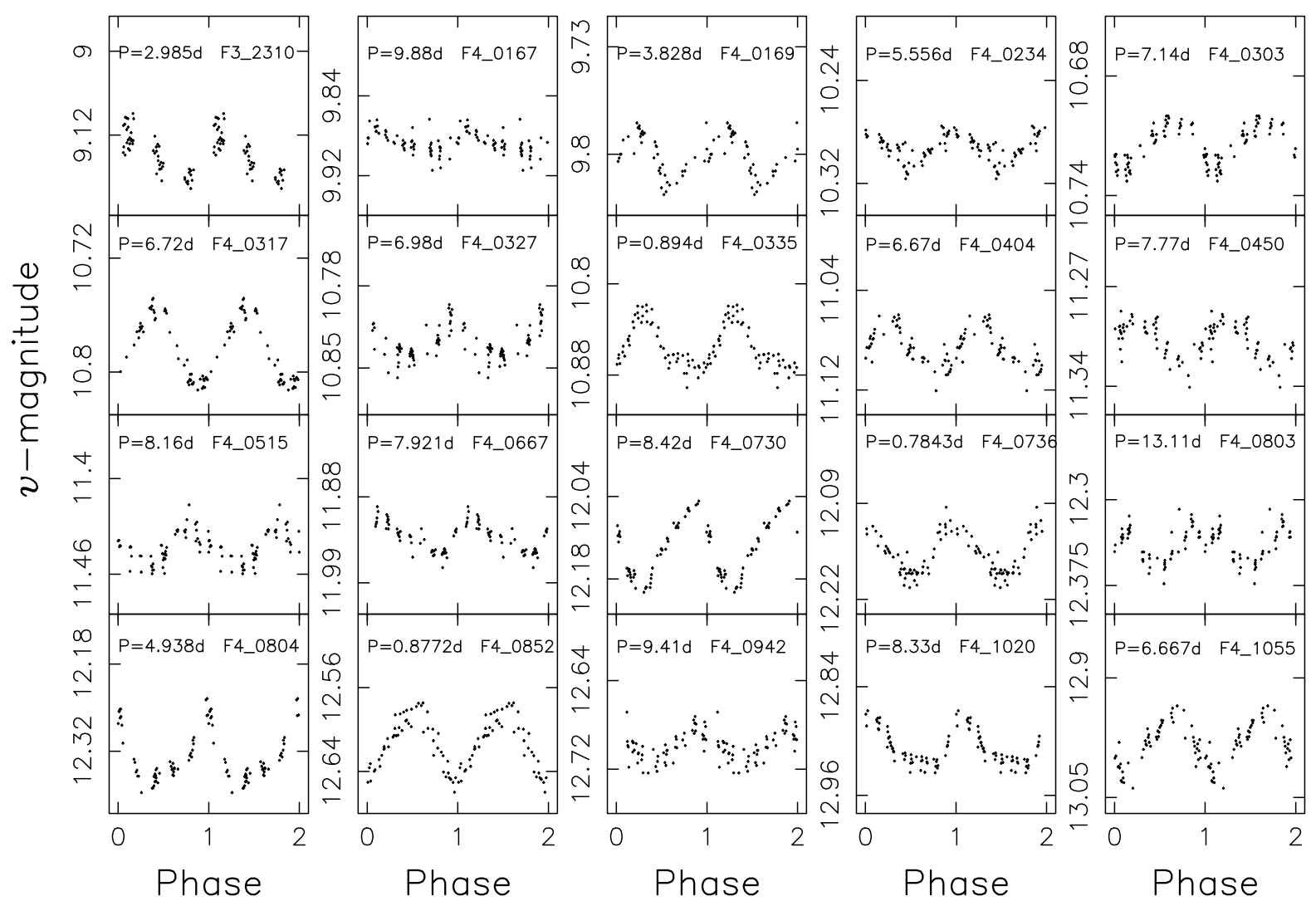

Fig. C.3. continued. 
A\&A 515, A100 (2010)

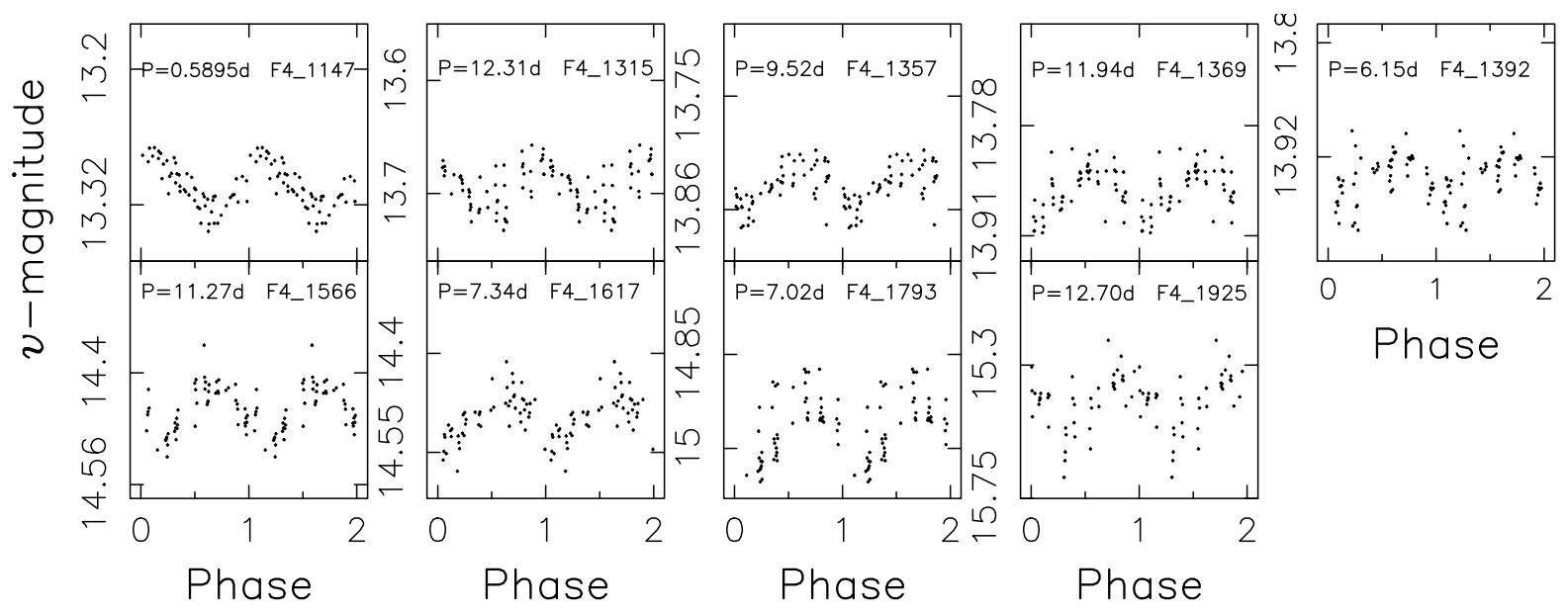

Fig. C.3. continued.

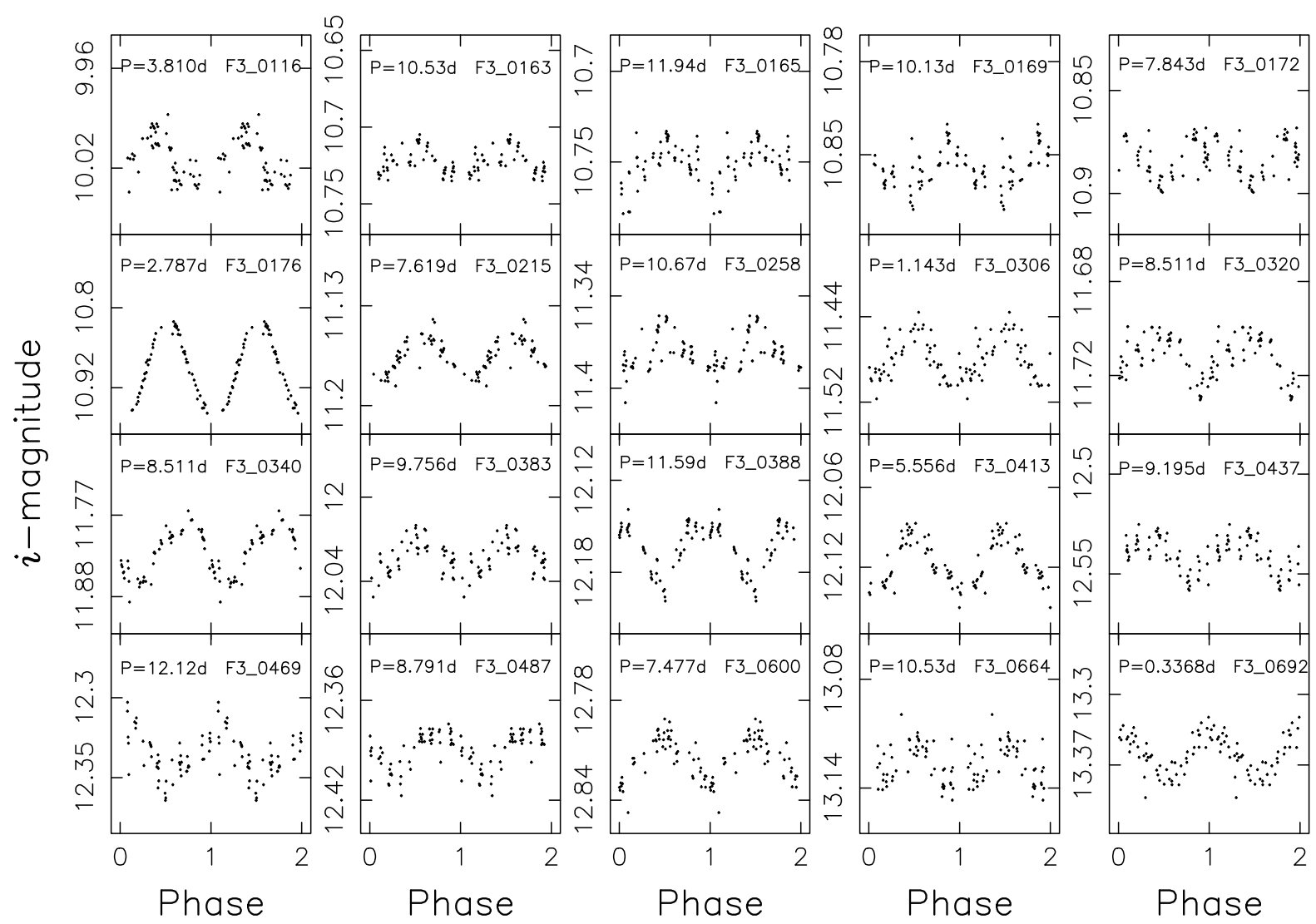

Fig. C.4. Period-phased photometric lightcurves for M 34 variables, derived from long-I observations. 
D. J. James et al.: The gyrochronology age of M 34

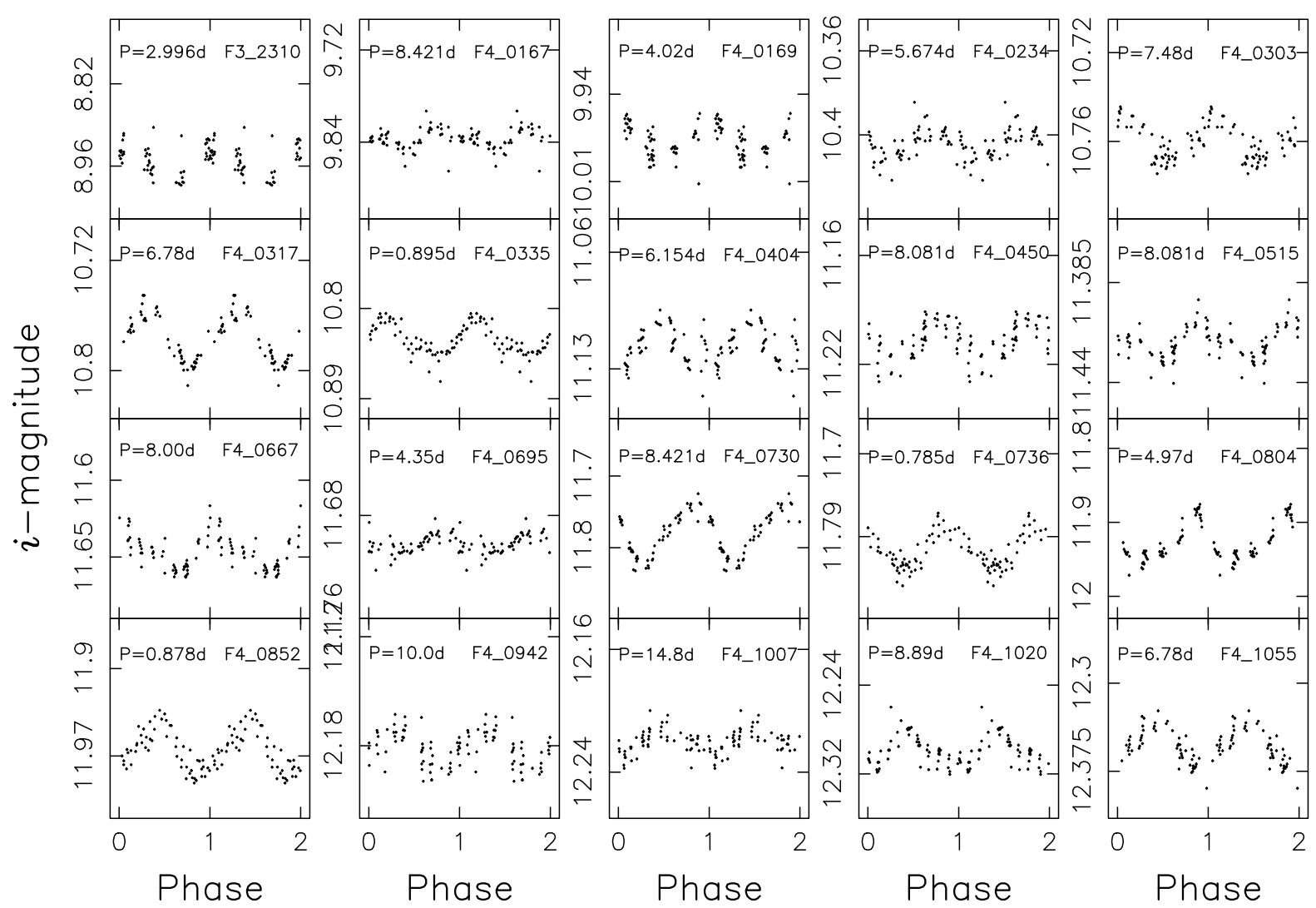

Fig. C.4. continued.
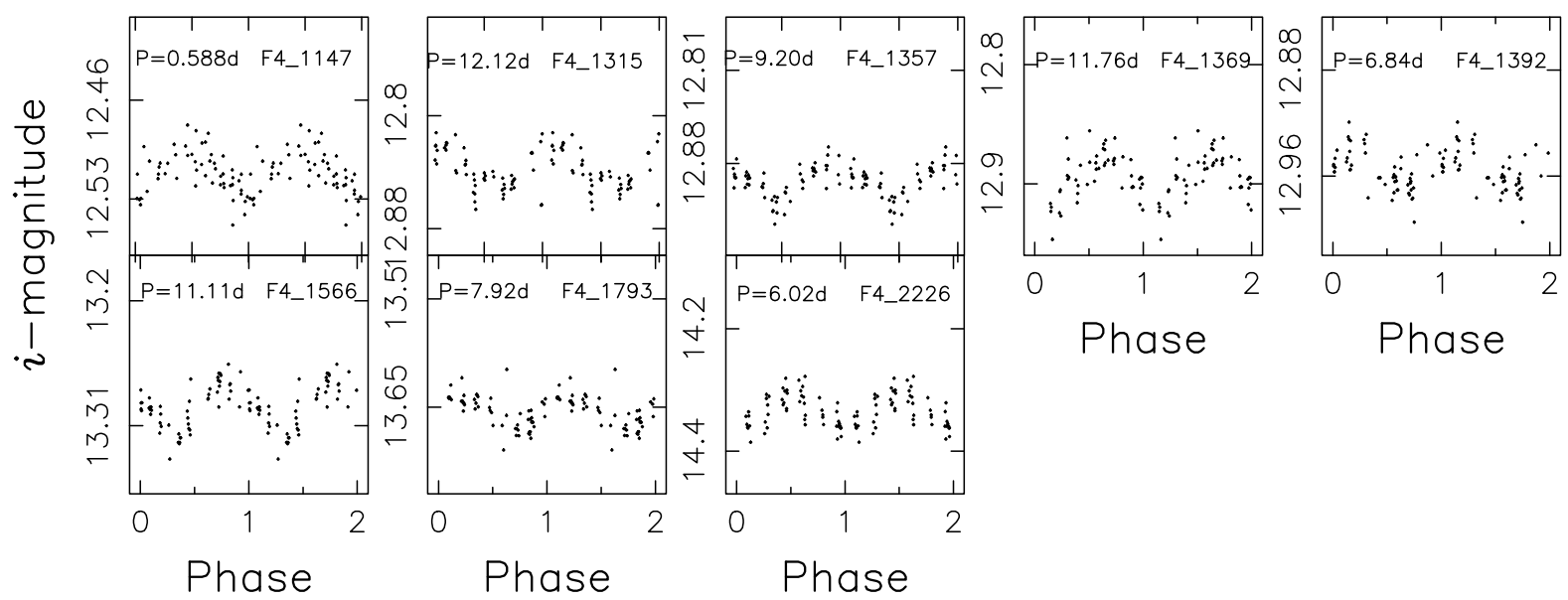

Fig. C.4. continued. 